DIVISION OR IMUTSMRIAL FYGIORT

OHIO STATF 3ORD OF FEALTH

ADI YOU IN BUSINESS FOR YOTP HFALM? 


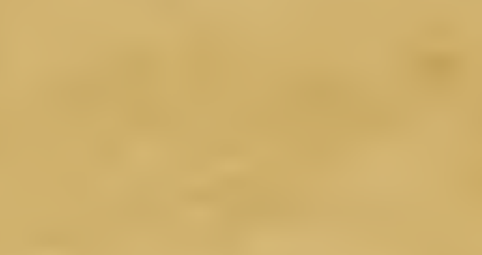

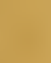






\section{Are You In Business For Your Health? IF NOT, YOU OUGHT TO BE}

A QUESTION WHICH MEANS

A Great Saving to Employer and Worker Alike

STANDARDS to be Observed in Developing Personal EFFICIENCY AND PRODUCTION

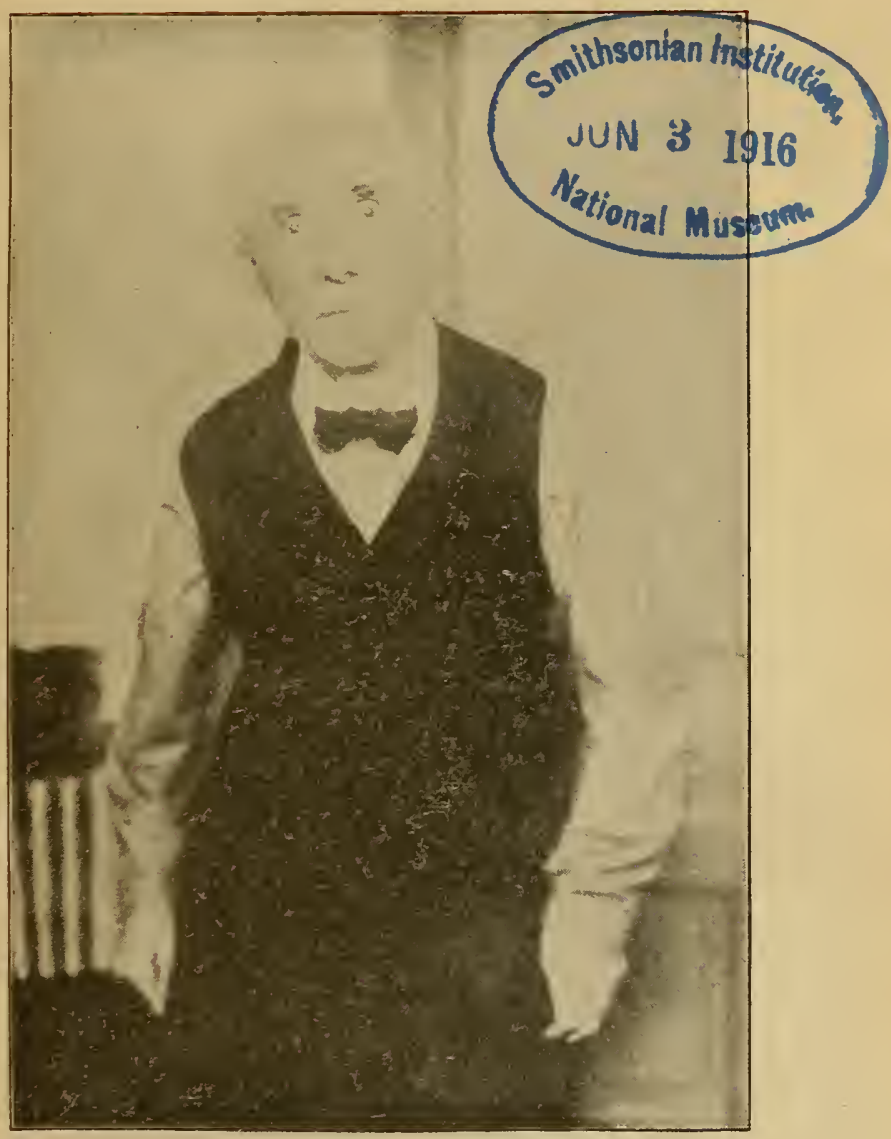

OLD AGE TWENTY YEARS TOO SOON

WHY?

Third (revised) edition. Issued March, 1916.

DIVISION OF INDUSTRIAL HYGIENE-OHIO STATE BOARD OF HEALTH

E. F. McCAMPBELL, Ph.D., M.D., Secretary and Executive Officer, Columbus, Ohio 


\section{THE SITUATION}

PREVENTABLE DISEASES take off $43 \%$ of all workers.

EXCEPT THE FARMER only 13\% of occupied persons reach the age of 70 years.

THE CHANCES OF LIVING as long as our fathers are steadily decreasing for all who have reached 40 years of age.

HEART DISEASES cause, in Ohio, one-seventh of all deaths. Over half occur before the allotted 70 years of life. One-fourth occur before 50 years of age. Deaths from Heart Diseases are increasing at the rate of over $5 \%$ annually.

ALL FORMS OF CHRONIC DISEASES are more PREVALENT in America than anywhere else in the civilized world. They are aiso INCREASING more rapidly in America than in any civilized country in the world.

\section{THE CAUSES}

I. Forgetting personal hygiene or the LAWS OF HEALTH in the midst of greater civilization.

II. Personal ignorance of ONE'S OWN SELF, as made known by physical examinations.

III. Presence of DANGERS IN THE SURROUNDINGS which cause sickness or add to sickness already present. These dangers are constantly increasing as time and civilization go on.

IV. No help when once down, or LACK OF INSURANCE PROTECTION.

\section{THE CURE}

I. LAWS OF HEALTH - Fifteen in Number: Consult "How to Live" by Fisher and Fisk,

1. Ventilate every room you occupy.

The Life Extension Institute, N. Y. City.

2. Wear lightweight, loose and porous clothes.

3. Get out of doors. Recreate, do not dissipate.

4. Breathe night-air at night time - open your bed-room windows.

5. Breathe deeply - 100 times each day.

6. Avoid over-eating and over-weight.

7. Eat sparingly of meat and eggs.

8. Eat some hard, some bulky and some raw foods, especially vegetables and fruits.

9. Eat the first three mouthfuls slowly. Eat the balance slowly. Drink water principally between meals.

10. Go to stool regularly and frequently.

11. Stand, sit and walk erect.

12. Do not allow poisons and infections to enter the body.

13. Keep the teeth, gums and tongue clean. Don't forget to clean them before going to bed.

14. Work, play, rest and sleep in moderation. Stop the pace which is killing the American race, especially lack of sleep.

15. Keep serene.

II. PHYSICAL EXAMINATIONS - Find Out How Nearly "All Right" You Are.

1. Remember that not one person in one hundred is physically perfect these days; for instance, your blood-pressure may be too high, or your heart action weak, or your kidneys affected, or your lungs "touched", all without signs which have seemed important to you.

2. Grasp the first chance for a Physical Examination. When you know for certain in what part you are not perfect, you will know what to do; and you will know also what not to do. "Know Thyself", said Socrates, 400 B. C., and we are just beginning to appreciate what the old sage meant. 


\section{Are You In Business For Your Health?}

IF NOT, YOU OUGHT TO BE

A QUESTION WHICH MEANS

A Great Saving to Employer and Worker Alike

STANDARDS to be Observed in Developing Personal EFFICIENCY AND PRODUCTIOWhoniantistitutio,

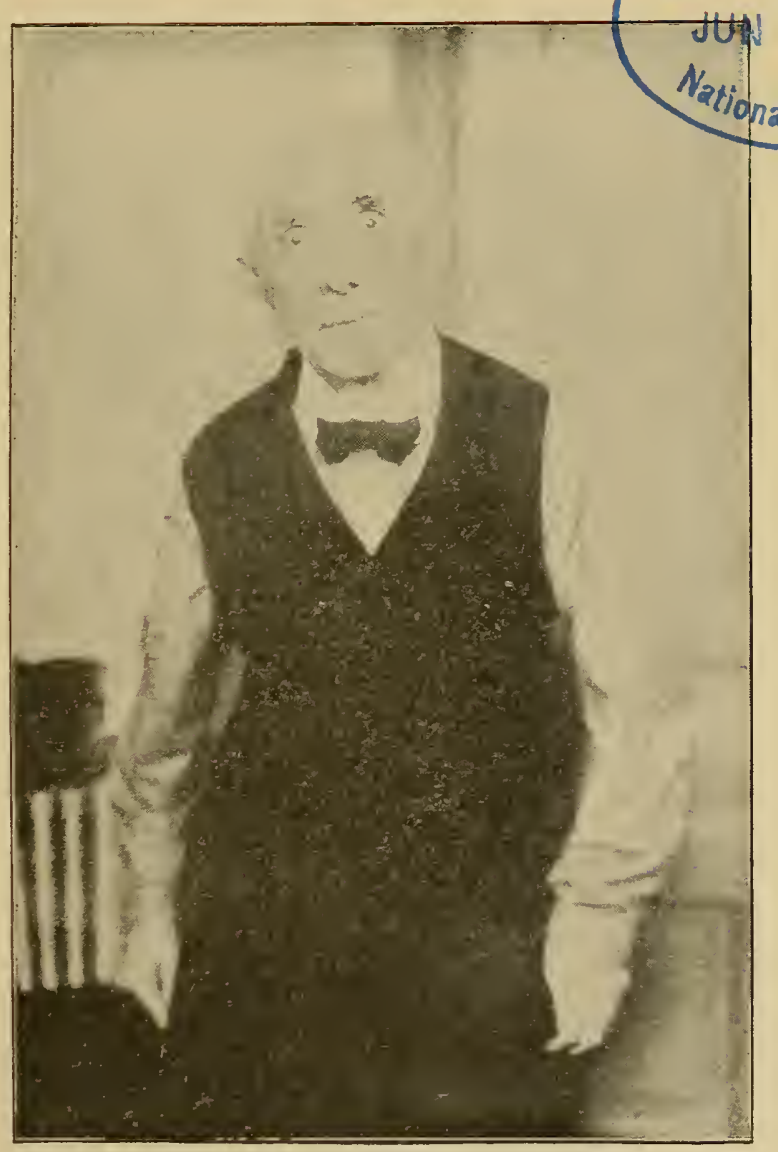

OLD AGE TWENTY YEARS TOO SOON

\section{WHY?}

Third (revised) edition. Issued March, 1916.

DIVISION OF INDUSTRIAL HYGIENE-OHIO STATE BOARD OF HEALTH

E. F. McCAMPBELL, Ph.D., M.D., Secretary and Executive Officer, Columbus, Ohio 


\section{THE SITUATION}

PREVENTABLE DISEASES take off $43 \%$ of all workers.

EXCEPT THE FARMER only $13^{\text {\% }}$ of of occupied persons reach the age of 70 years.

THE CHANCES OF LIVING as long as our fathers are steadily decreasing for all who have reached 40 years of age.

HEART DISEASES cause, in Ohio, one-seventh of all deaths. Over half occur before the allotted 70 years of life. One-fourth occur before 50 years of age. Deaths from Heart Diseases are increasing at the rate of over $5 \%$ annually.

ALL FORMS OF CHRONIC DISEASES are more PREVALENT in America than anywhere else in the civilized world. They are aiso INCREASING more rapidly in America than in any civilized country in the world.

\section{THE CAUSES}

I. Forgetting personal hygiene or the LAWS OF HEALTH in the midst of greater civilization.

II. Personal ignorance of ONE'S OWN SELF, as made known by physical examinations.

III. Presence of DANGERS IN THE SURROUNDINGS which cause sickness or add to sickness already present. These dangers are constantly increasing as time and civilization go on.

IV. No help when once down, or LACK OF INSURANCE PROTECTION.

\section{THE CURE}

I. LAWS OF HEALTH - Fifteen in Number: Consult "How to Live" by Fisher and Fisk,

1. Ventilate every room you occupy.

The Life Extension Institute, N. Y. City.

2. Wear lightweight, loose and porous clothes.

3. Get out of doors. Recreate, do not dissipate.

4. Breathe night-air at night time - open your bed-room windows.

5. Breathe deeply -100 times each day.

6. Avoid over-eating and over-weight.

7. Eat sparingly of meat and eggs.

8. Eat some hard, some bulky and some raw foods, especially vegetables and fruits.

9. Eat the first three mouthfuls slowly. Eat the balance slowly. Drink water principally between meals.

10. Go to stool regularly and frequently.

11. Stand, sit and walk erect.

12. Do not allow poisons and infections to enter the body.

13. Keep the teeth, gums and tongue clean. Don't forget to clean them before going to bed.

14. Work, play, rest and sleep in moderation. Stop the pace which is killing the American race, especially lack of sleep.

15. Keep serene.

II. PHYSICAL EXAMINATIONS - Find Out How Nearly “All Right" You Áre.

1. Remember that not one person in one hundred is physically perfect these days; for instance, your blood-pressure may be too high, or your heart action weak, or your kidneys affected, or your lungs "touched", all without signs which have seemed important to you.

2. Grasp the first chance for a Physical Examination. When you know for certain in what part you are not perfect, you will know what to do; and you will know also what not to do. "Know Thyself", said Socrates, 400 B. C., and we are just beginning to appreciate what the old sage meant. 


\section{Are You In Business For Your Health?}

IF NOT, YOU OUGHT TO BE

A QUESTION WHICH MEANS

A Great Saving to Employer and Worker Alike

STANDARDS to be Observed in Developing Personal EFFICIENCY AND PRODUCTION

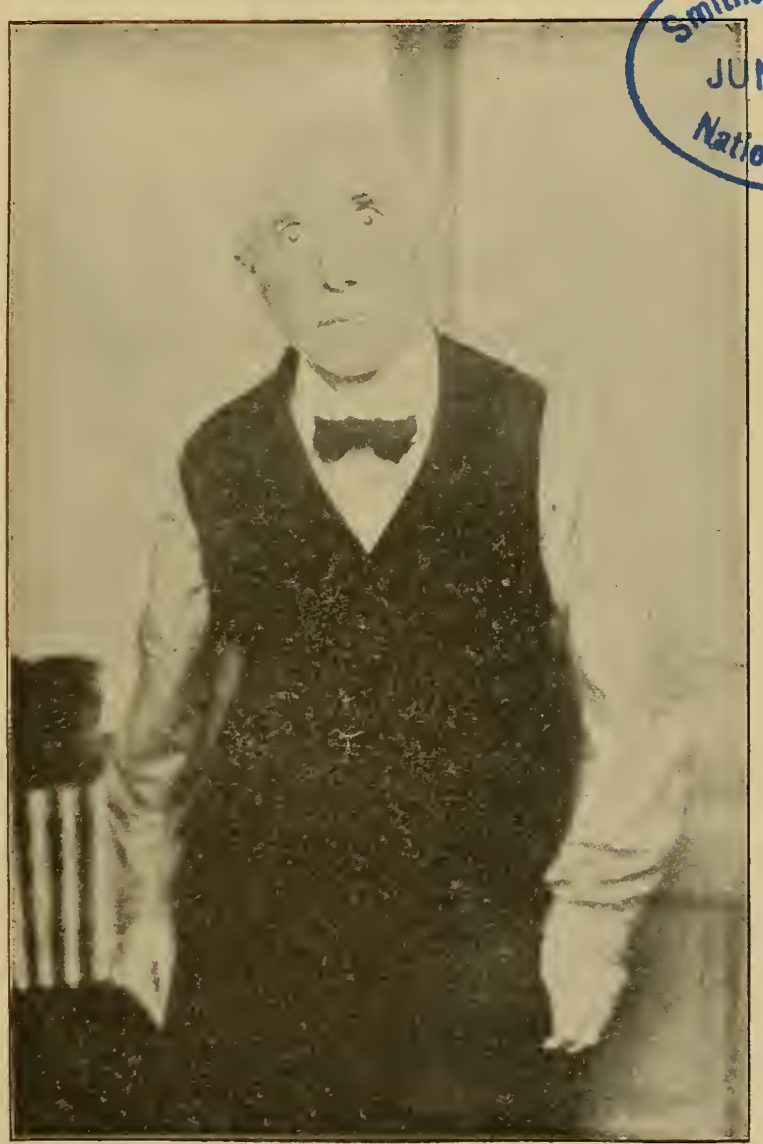

OLD AGE TWENTY YEARS TOO SOON

WHY?

Third (revised) edition. Issued March, 1916.

DIVISION OF INDUSTRIAL HYGIENE-OHIO STATE BOARD OF HEALTH

E. F. McCAMPBELL, Ph.D., M.D., Secretary and Executive Officer, Columbus, Ohio 


\section{THE SITUATION}

PREVENTABLE DISEASES take off $43 \%$ of all workers.

EXCEPT THE FARMER only $13 \%$ of occupied persons reach the age of 70 years.

THE CHANCES OF LIVING as long as our fathers are steadily decreasing for all who have reached 40 years of age.

HEART DISEASES cause, in Ohio, one-seventh of all deaths. Over half occur before the allotted 70 years of life. One-fourth occur before 50 years of age. Deaths from Heart Diseases are increasing at the rate of over $5 \%$ annually.

ALL FORMS OF CHRONIC DISEASES are more PREVALENT in America than anywhere else in the civilized world. They are aiso INCREASING more rapidly in America than in any civilized country in the world.

\section{THE CAUSES}

I. Forgetting personal hygiene or the LAWS OF HEALTH in the midst of greater civilization.

II. Personal ignorance of ONE'S OWN SELF, as made known by physical examinations.

III. Presence of DANGERS IN THE SURROUNDINGS which cause sickness or add to sickness already present. These dangers are constantly increasing as time and civilization go on.

IV. No help when once down, or LACK OF INSURANCE PROTECTION.

\section{THE CURE}

I. LAWS OF HEALTH - Fif teen in Number : Consult "How to Live" by Fisher and Fisk,

1. Ventilate every room you occupy.

The Life Extension Institute, N. Y. City.

2. Wear lightweight, loose and porous clothes.

3. Get out of doors. Recreate, do not dissipate.

4. Breathe night-air at night time - open your bed-room windows.

5. Breathe deeply - 100 times each day.

6. Avoid over-eating and over-weight.

7. Eat sparingly of meat and eggs.

8. Eat some hard, some bulky and some raw foods, especially vegetables and fruits.

9. Eat the first three mouthfuls slowly. Eat the balance slowly. Drink water principally between meals.

10. Go to stool regularly and frequently.

11. Stand, sit and walk erect.

12. Do not allow poisons and infections to enter the body.

13. Keep the teeth, gums and tongue clean. Don't forget to clean them before going to bed.

14. Work, play, rest and sleep in moderation. Stop the pace which is killing the American race, especially lack of sleep.

15. Keep serene.

II. PHYSICAL EXAMINATIONS - Find Out How Nearly "All Right" You Are.

1. Remember that not one person in one hundred is physically perfect these days; for instance, your blood-pressure may be too high, or your heart action weak, or your kidneys affected, or your lungs "touched", all without signs which have seemed important to you.

2. Grasp the first chance for a Physical Examination. When you know for certain in what part you are not perfect, you will know what to do; and you will know also what not to do. "Know Thyself", said Socrates, 400 B. C., and we are just beginning to appreciate what the old sage meant. 


\section{Are You In Business For Your Health?}

IF NOT, YOU OUGHT TO BE

A QUESTION WHICH MEANS

A Great Saving to Employer and Worker Alike

STANDARDS to be Observed in Developing Personal

EFFICIENCY AND PRODUCTION

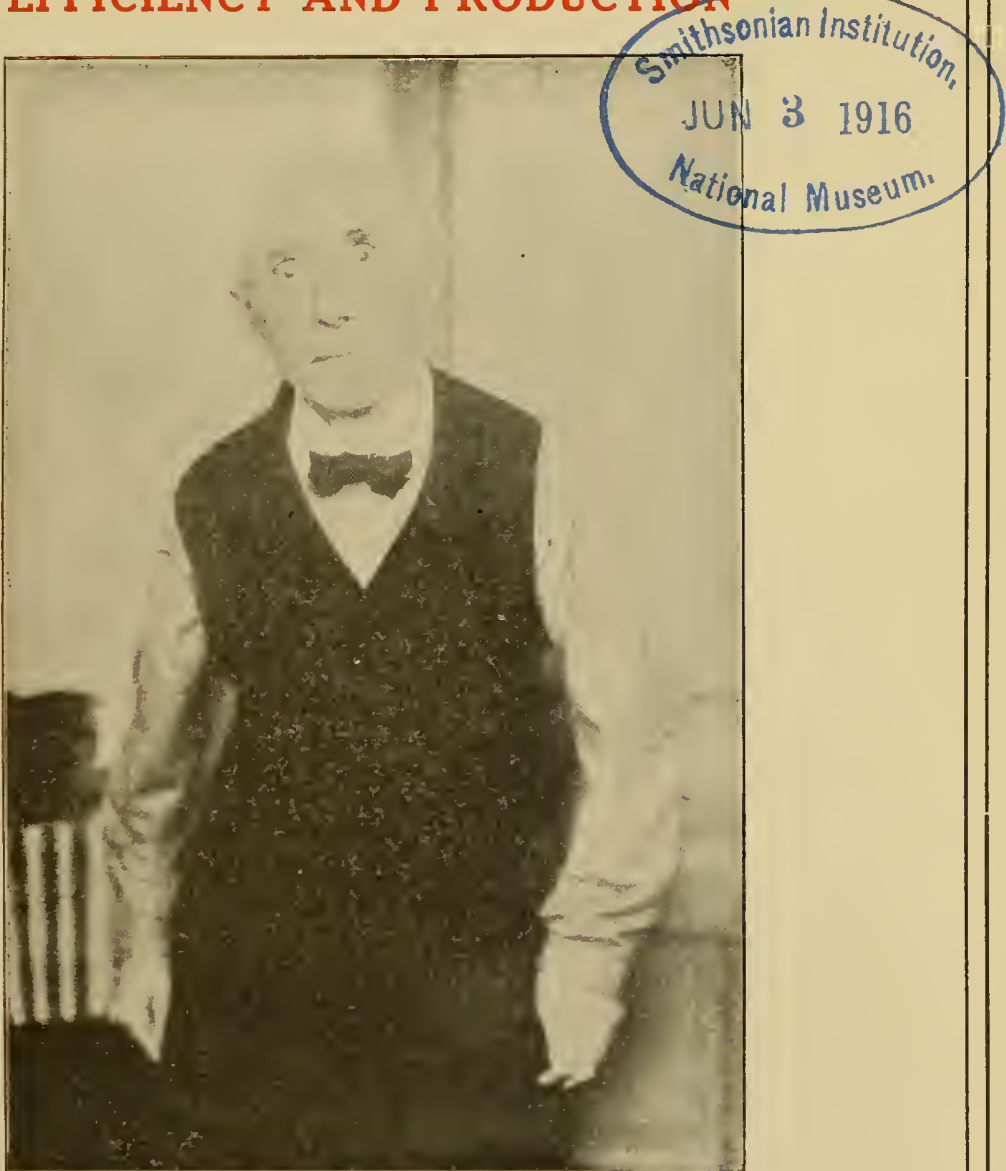

OLD AGE TWENTY YEARS TOO SOON

\section{WHY?}

Third (revised) edition. Issued March, 1916.

DIVISION OF INDUSTRIAL HYGIENE-OHIO STATE BOARD OF HEALTH

E. F. McCAMPBELL, Ph.D., M.D., Secretary and Executive Officer, Columbus, Ohio 


\section{THE SITUATION}

PREVENTABLE DISEASES take off $43 \%$ of all workers.

EXCEPT THE FARMER only $13 \%$ of occupied persons reach the age of 70 years. THE CHANCES OF LIVING as long as our fathers are steadily decreasing for all who have reached 40 years of age.

HEART DISEASES cause, in Ohio, one-seventh of all deaths. Over half occur before the allotted 70 years of life. One-fourth occur before 50 years of age. Deaths from Heart Diseases are increasing at the rate of over $5 \%$ annually.

ALL FORMS OF CHRONIC DISEASES are more PREVALENT in America than anywhere else in the civilized world. They are also INCREASING more rapidly in America than in any civilized country in the world.

\section{THE CAUSES}

I. Forgetting personal hygiene or the LAWS OF HEALTH in the midst of greater civilization.

II. Personal ignorance of ONE'S OWN SELF, as made known by physical examinations.

III. Presence of DANGERS IN THE SURROUNDINGS which cause sickness or add to sickness already present. These dangers are constantly increasing as time and civilization go on.

IV. No help when once down, or LACK OF INSURANCE PROTECTION.

\section{THE CURE}

I. LAWS OF HEALTH - Fifteen in Number: Consult "How to Live" by Fisher and Fisk,

1. Ventilate every room you occupy.

The Life Extension Institute, N. Y. City.

2. Wear lightweight, loose and porous clothes.

3. Get out of doors. Recreate, do not dissipate.

4. Breathe night-air at night time - open your bed-room windows.

5. Breathe deeply - 100 times each day.

6. Avoid over-eating and over-weight.

7. Eat sparingly of meat and eggs.

8. Eat some hard, some bulky and some raw foods, especially vegetables and fruits.

9. Eat the first three mouthfuls slowly. Eat the balance slowly. Drink water principally between meals.

10. Go to stool regularly and frequently.

11. Stand, sit and walk erect.

12. Do not allow poisons and infections to enter the body.

13. Keep the teeth, gums and tongue clean. Don't forget to clean them before going to bed.

14. Work, play, rest and sleep in moderation. Stop the pace which is killing the American race, especially lack of sleep.

15. Keep serene.

II. PHYSICAL EXAMINATIONS - Find Out How Nearly "All Right" You Are.

1. Remember that not one person in one hundred is physically perfect these days; for instance, your blood-pressure may be too high, or your heart action weak, or your kidneys affected, or your lungs "touched", all without signs which have seemed important to you.

2. Grasp the first chance for a Physical Examination. When you know for certain in what part you are not perfect, you will know what to do; and you will know also what not to do. "Know Thyself", said Socrates, 400 B. C., and we are just beginning to appreciate what the old sage meant. 


\section{Are You In Business For Your Health?}

IF NOT, YOU OUGHT TO BE

A QUESTION WHICH MEANS

A Great Saving to Employer and Worker Alike

STANDARDS to be Observed in Developing Personal EFFICIENCY AND PRODUCTION

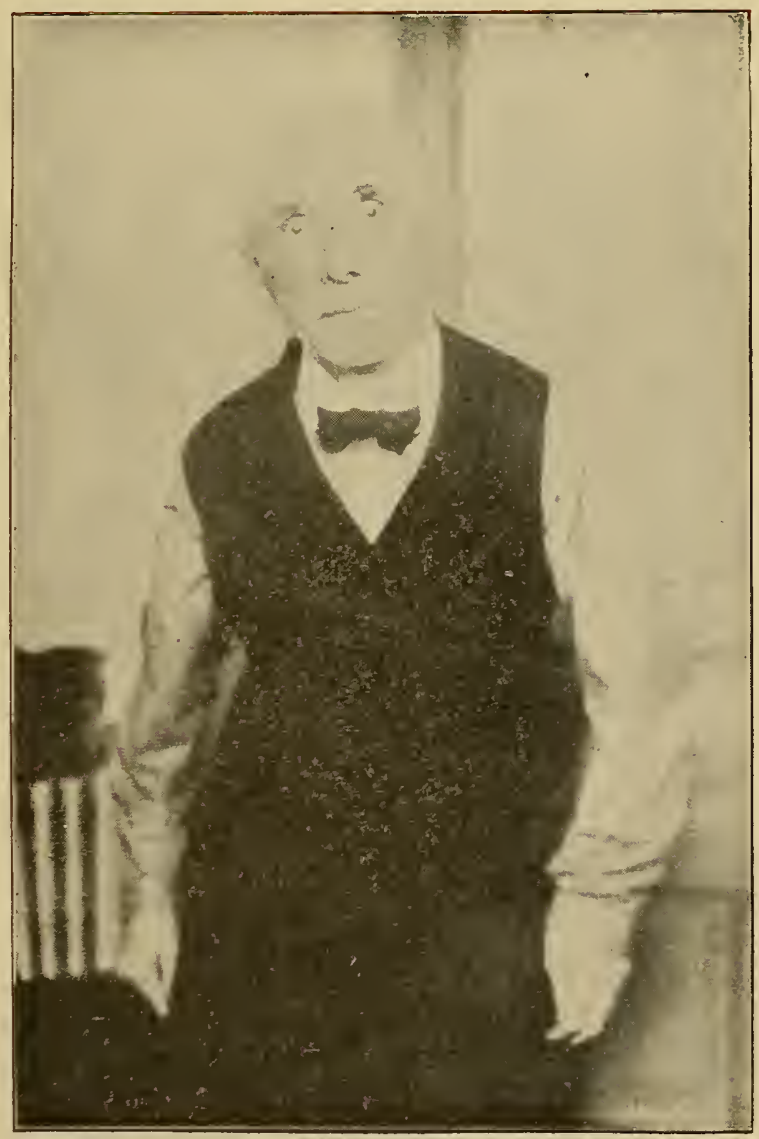

OLD AGE TWENTY YEARS TOO SOON

\section{WHY?}

Third (revised) edition. Issued March, 1916.

DIVISION OF INDUSTRIAL HYGIENE-OHIO STATE BOARD OF HEALTH

E. F. McCAMPBELL, Ph.D., M.D., Secretary and Executive Officer, Columbus, Ohio 


\section{THE SITUATION}

PREVENTABLE DISEASES take off $43 \%$ of all workers.

EXCEPT THE FARMER only 13\% of occupied persons reach the age of 70 years.

THE CHANCES OF LIVING as long as our fathers are steadily decreasing for all who have reached 40 years of age.

HEART DISEASES cause, in Ohio, one-seventh of all deaths. Over half occur before the allotted 70 years of life. One-fourth occur before 50 years of age. Deaths from Heart Diseases are increasing at the rate of over $5 \%$ annually.

ALL FORMS OF CHRONIC DISEASES are more PREVALENT in America than anywhere else in the civilized world. They are aiso INCREASING more rapidly in America than in any civilized country in the world.

\section{THE CAUSES}

I. Forgetting personal hygiene or the LAWS OF HEALTH in the midst of greater civilization.

II. Personal ignorance of ONE'S OWN SELF, as made known by physical examinations.

III. Presence of DANGERS IN THE SURROUNDINGS which cause sickness or add to sickness already present. These dangers are constantly increasing as time and civilization go on.

IV. No help when once down, or LACK OF INSURANCE PROTECTION.

\section{THE CURE}

I. LAWS OF HEALTH - Fifteen in Number: Consult "How to Live" by Fisher and Fisk,

1. Ventilate every room you occupy.

The Life Extension Institute, N. Y. City.

2. Wear lightweight, loose and porous clothes.

3. Get out of doors. Recreate, do not dissipate.

4. Breathe night-air at night time - open your bed-room windows.

5. Breathe deeply - 100 times each day.

6. Avoid over-eating and over-weight.

7. Eat sparingly of meat and eggs.

8. Eat some hard, some bulky and some raw foods, especially vegetables and fruits.

9. Eat the first three mouthfuls slowly. Eat the balance slowly. Drink water principally between meals.

10. Go to stool regularly and frequently.

11. Stand, sit and walk erect.

12. Do not allow poisons and infections to enter the body.

13. Keep the teeth, gums and tongue clean. Don't forget to clean them before going to bed.

14. Work, play, rest and sleep in moderation. Stop the pace which is killing the American race, especially lack of sleep.

15. Keep serene.

II. PHYSICAL EXAMINATIONS - Find Out How Nearly "All Right" You Are.

1. Remember that not one person in one hundred is physically perfect these days; for instance, your blood-pressure may be too high, or your heart action weak, or your kidneys affected, or your lungs "touched", all without signs which have seemed important to you.

2. Grasp the first chance for a Physical Examination. When you know for certain in what part you are not perfect, you will know what to do; and you will know also what not to do. "Know Thyself", said Socrates, 400 B. C., and we are just beginning to appreciate what the old sage meant. 


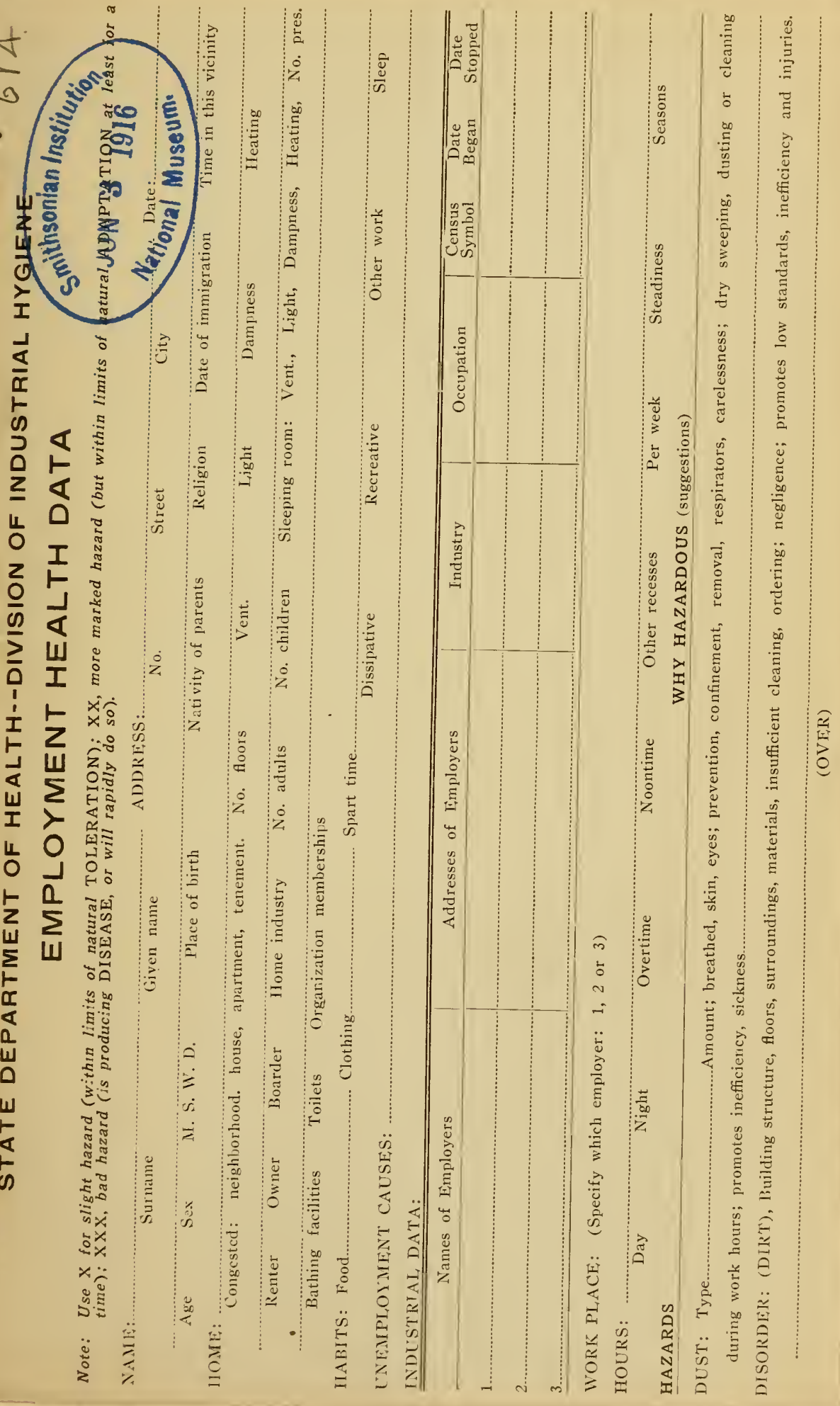




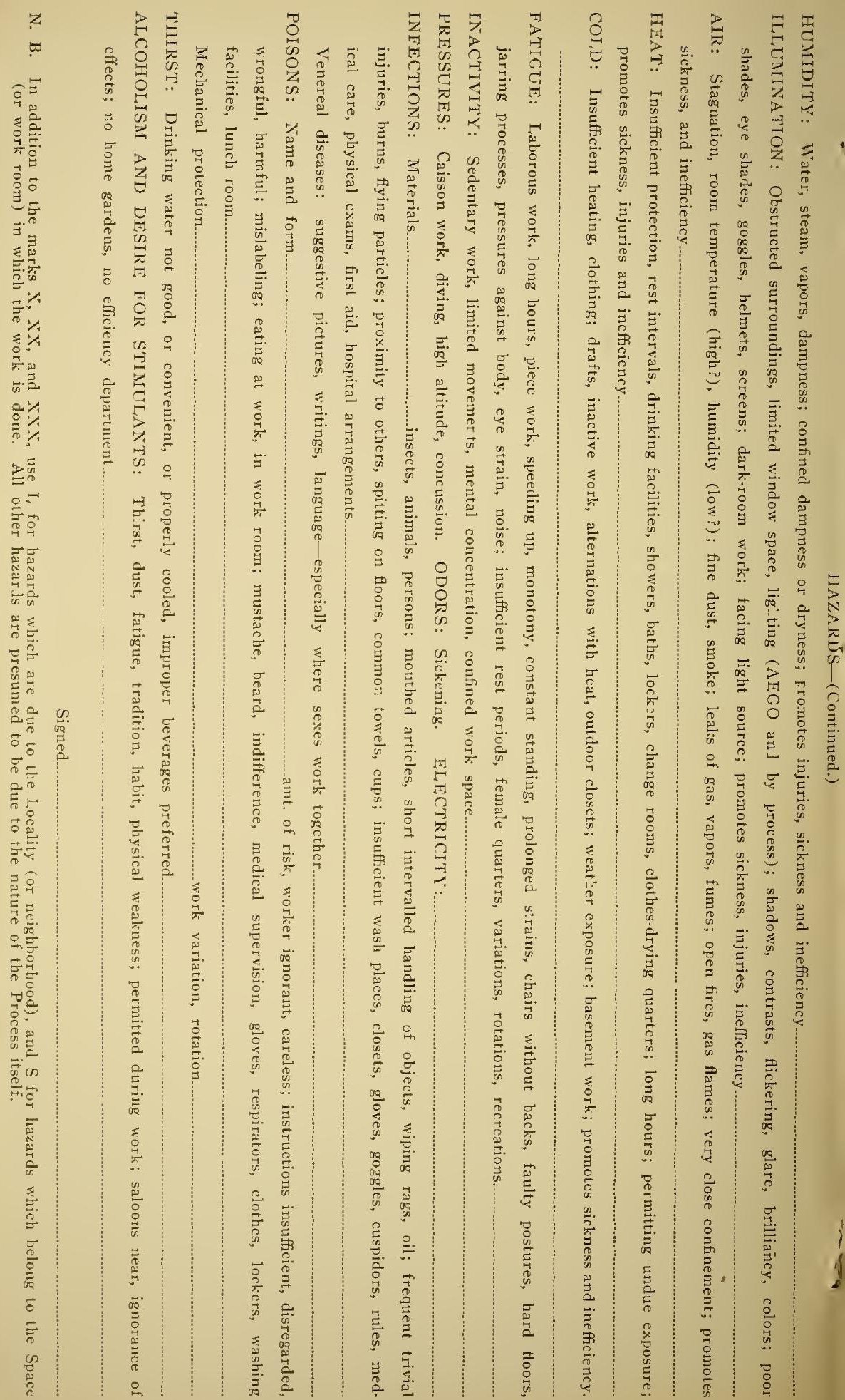




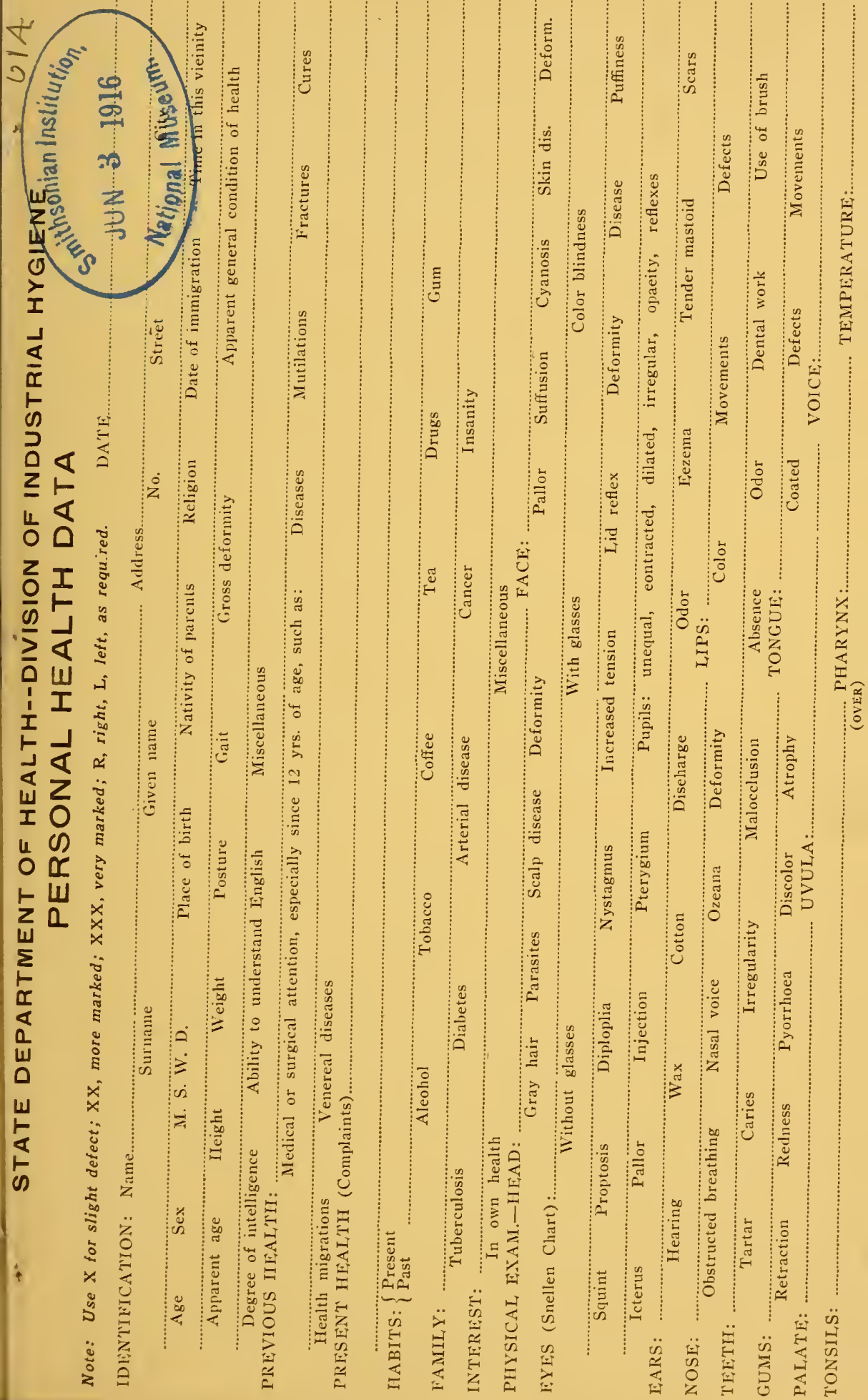




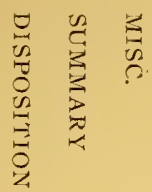

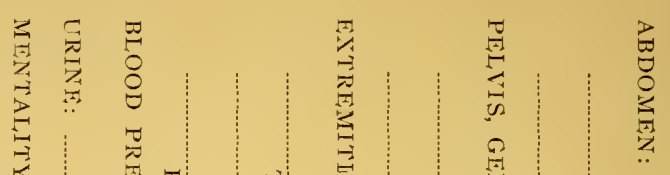

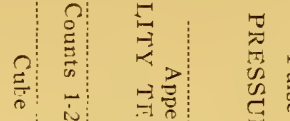

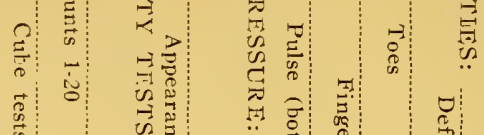$$
\text { 吾: }
$$

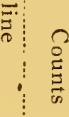$$
\forall \hat{\imath}
$$

$\therefore \bar{b}$

窟常焉

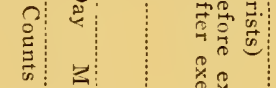

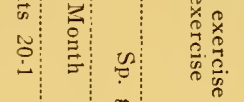

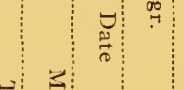

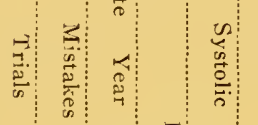

$\begin{array}{l:c} & 0 \\ 0 & 5 \\ 0 & 5 \\ 0 & 0 \\ 0 & 0 \\ 0 & 0\end{array}$

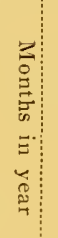

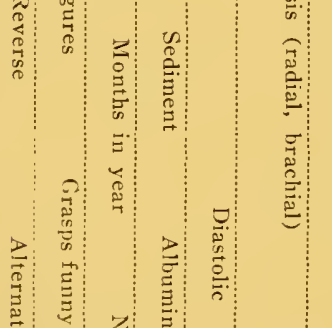

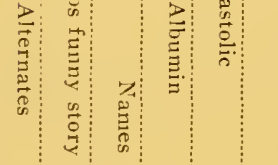

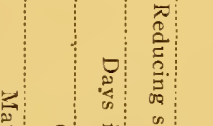

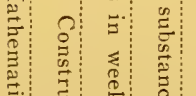

ڤ.

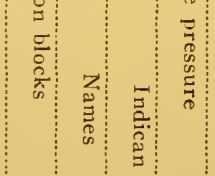

I

永:

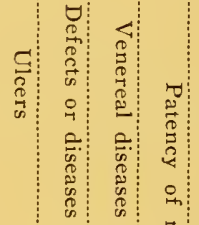

苛

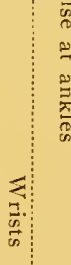

竞

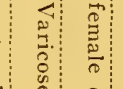

年

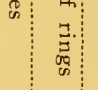

की $\stackrel{0}{\vec{E}: ~}$

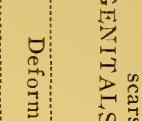

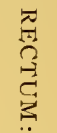

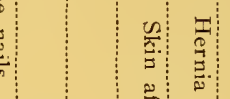

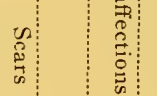

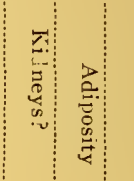

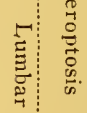

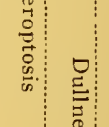

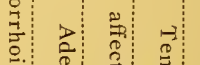

焉

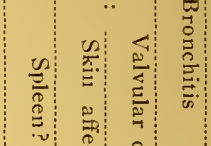

畓:

क

0
0
7
7

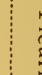

t:

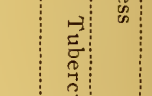

a

定

है:

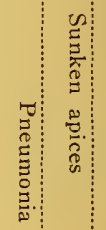

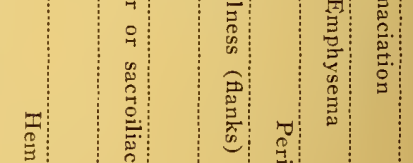

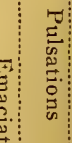

ב.

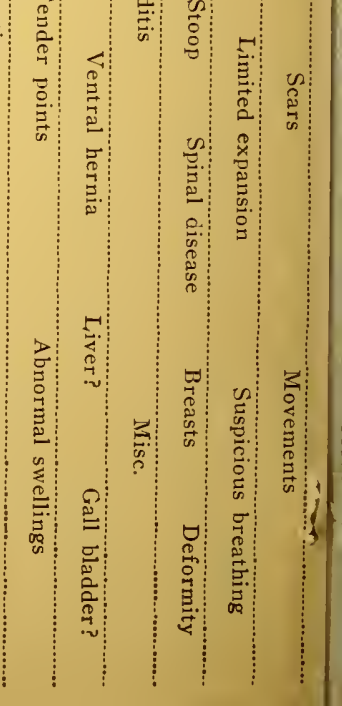




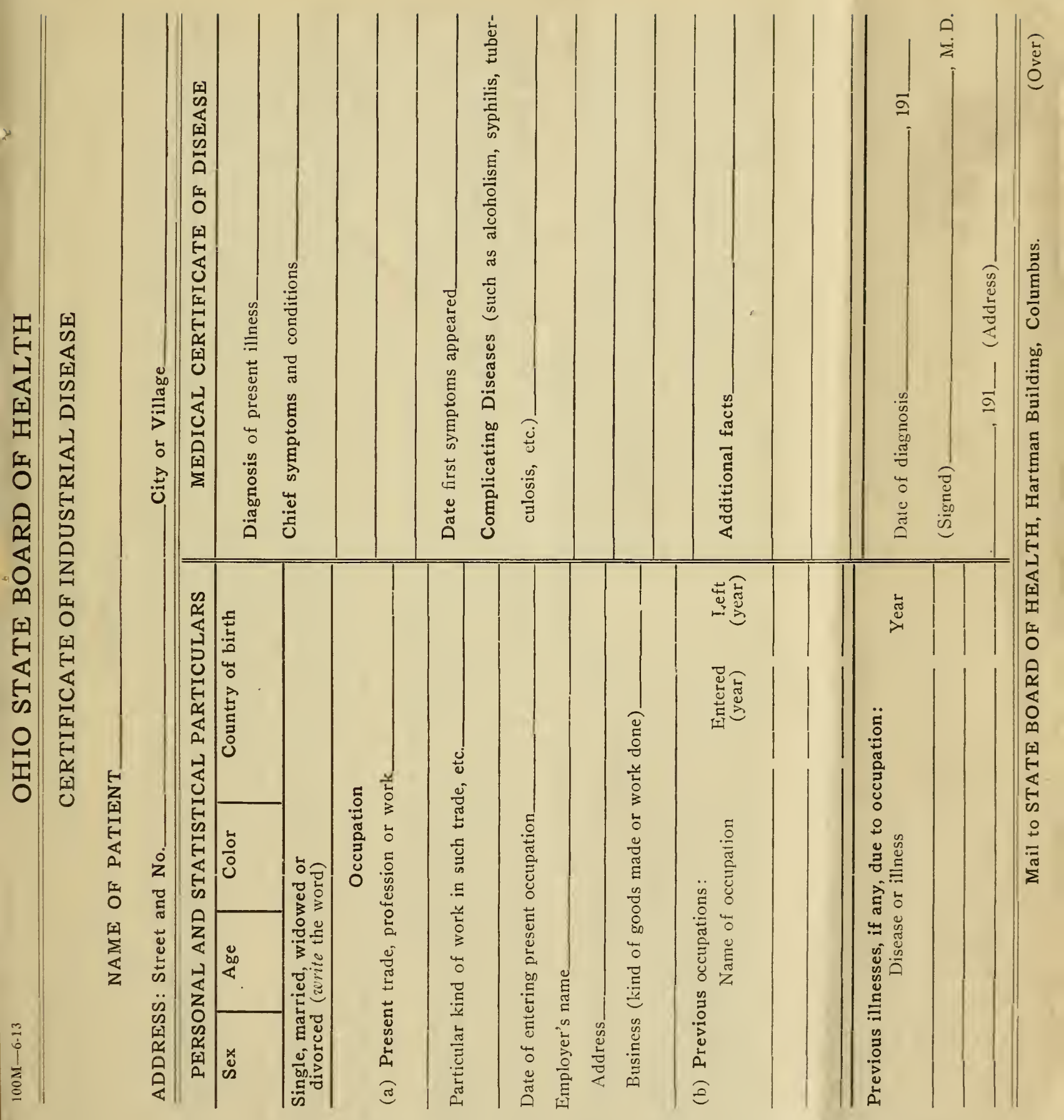

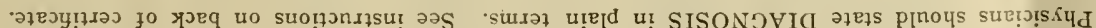

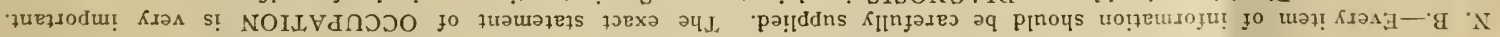

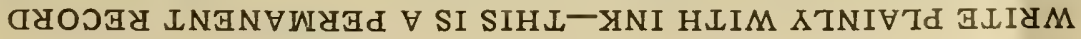




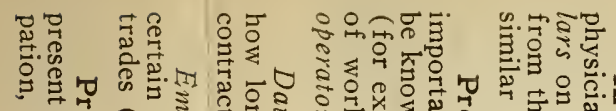

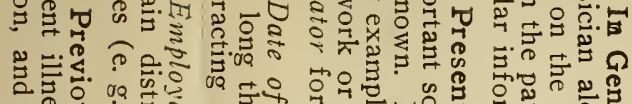
. \&

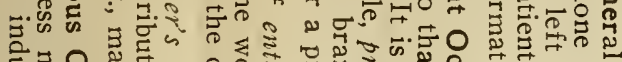
Е

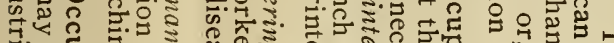

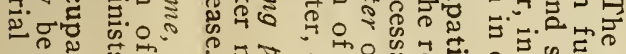
年

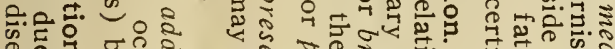

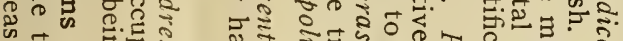

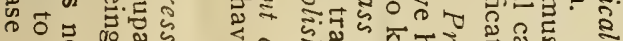

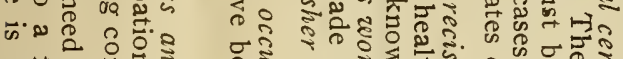

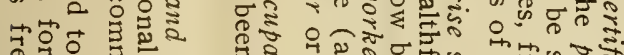

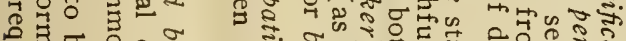

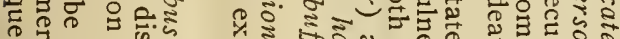

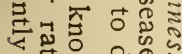

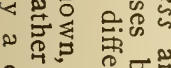

政

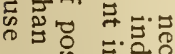

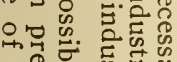

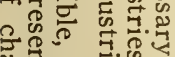

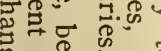

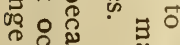

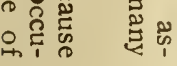

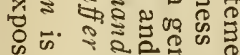

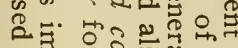

of

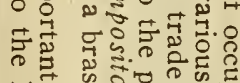

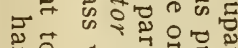

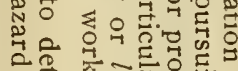

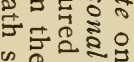

9

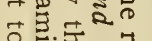

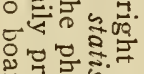

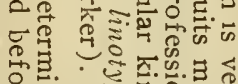

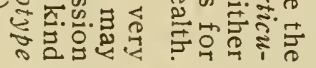

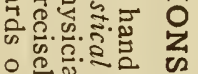

$\rightarrow 0$

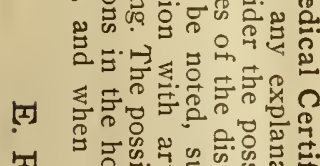

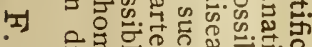

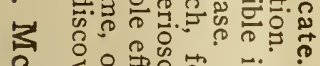

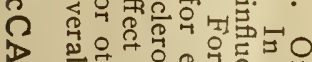

$\rightarrow \quad \begin{aligned} & 0 \\ & 0\end{aligned}$

T

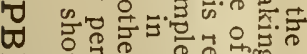

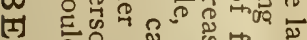

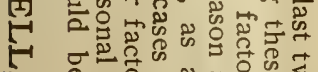

年

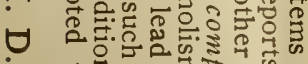

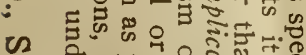

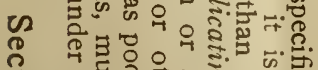

望

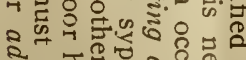

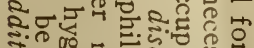

苛

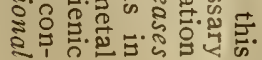

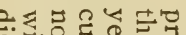

롤유 을

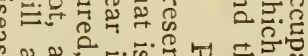

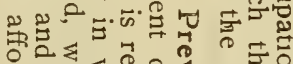

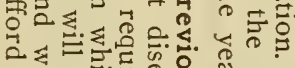

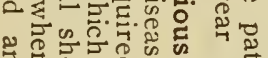

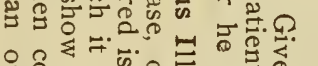

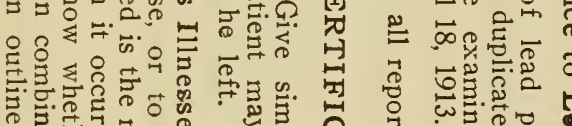

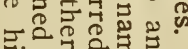

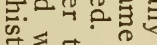

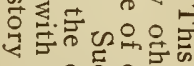

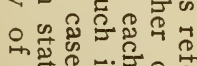

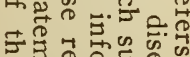

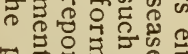

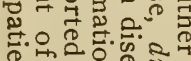

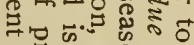

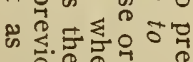

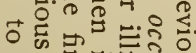

$\circ$ 出

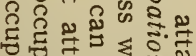

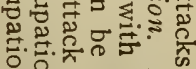

它.号.

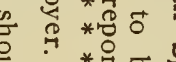

§。

通

宽鲂

迅
总总 号

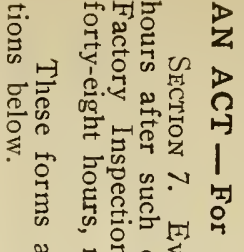

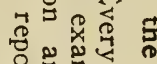

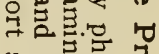

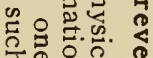

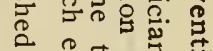

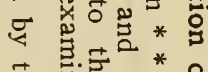

穴 哥穴者*

थ 设 *0

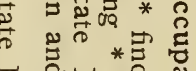

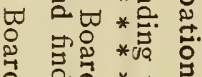

․ㅡㄹ

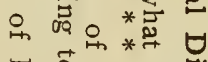

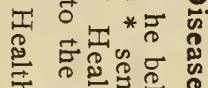

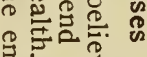

芑哭必总

* *

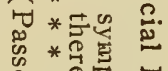

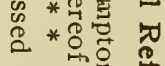

볼

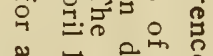

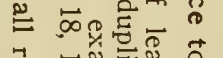

总武

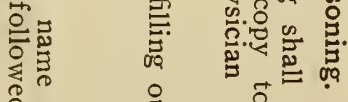

चㅇ

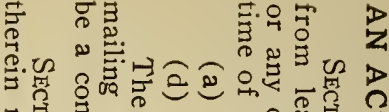

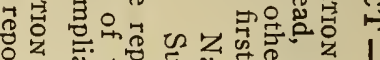

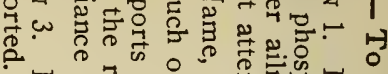

ॠ

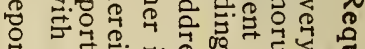

क

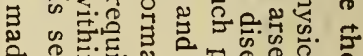

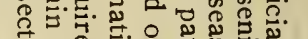

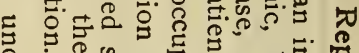

号 密

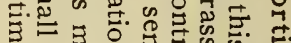

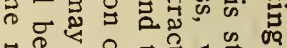

궁휴 웅

듬

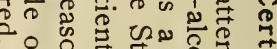

ఈ 寻

ค인

क다

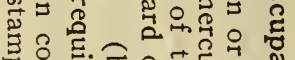

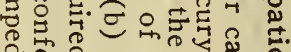

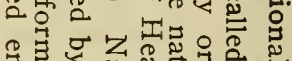

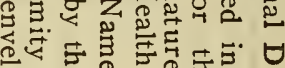

5 $\Omega$ क

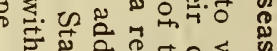

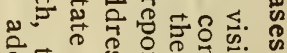

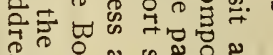

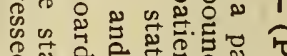

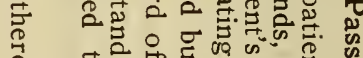

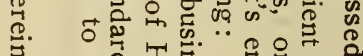

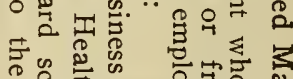

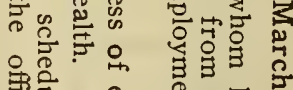

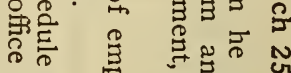

$\Omega$ [I]

点

II

T11

[स]

D

的 


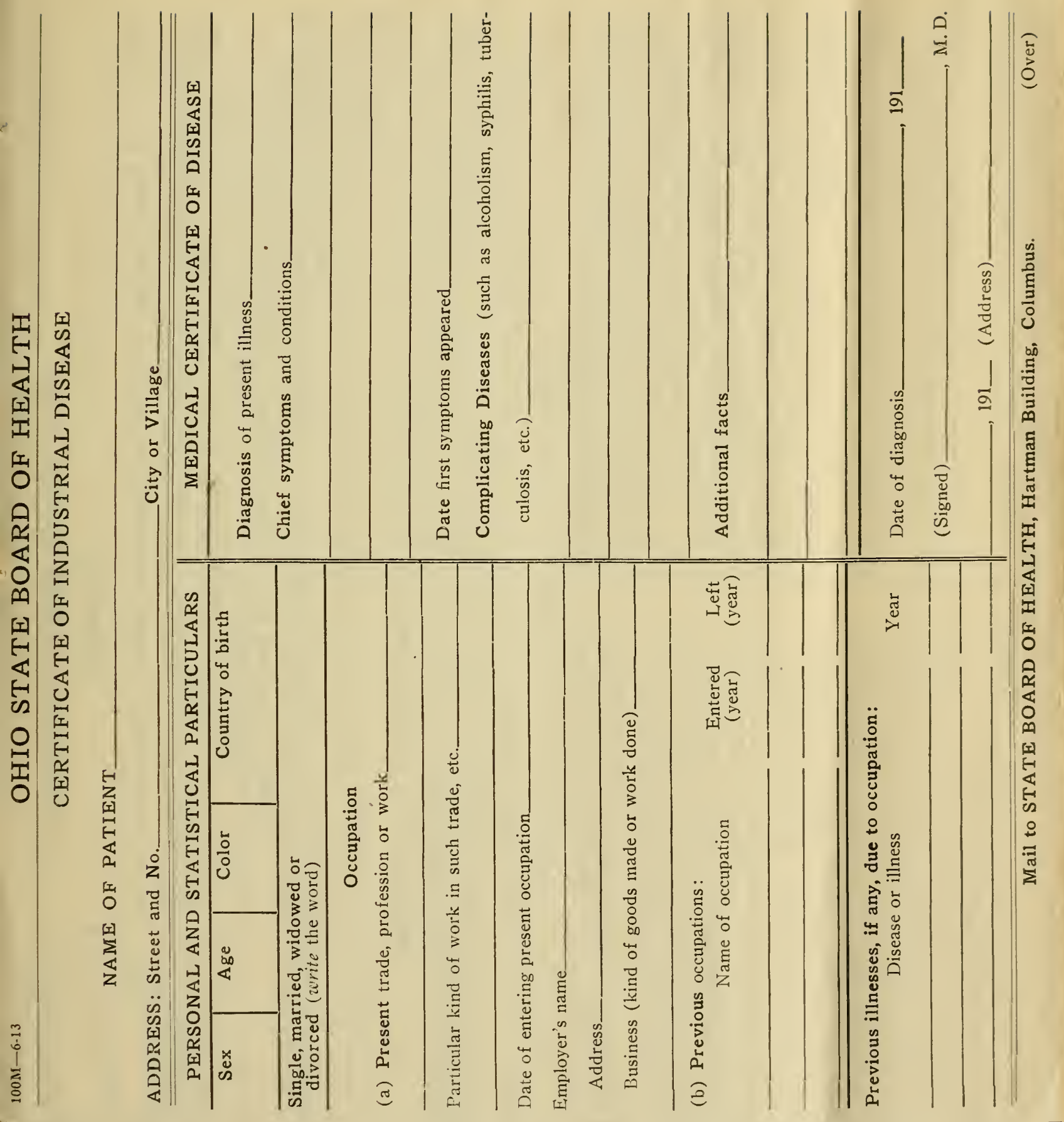

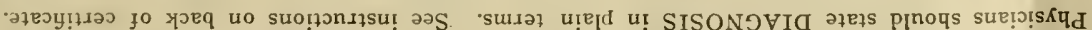

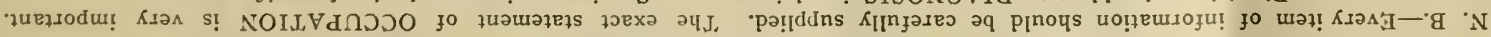




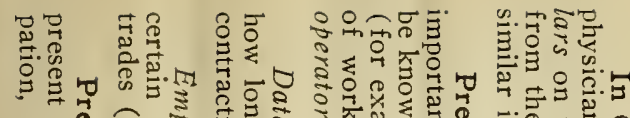
a res

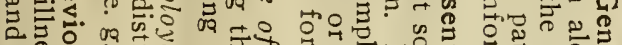

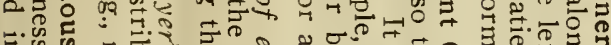

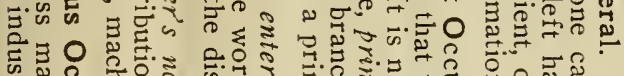

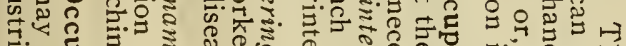

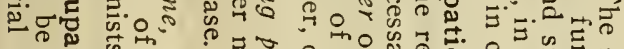

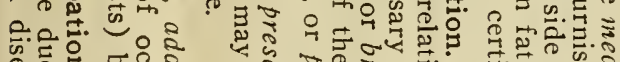

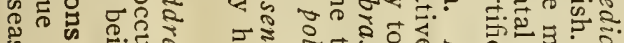

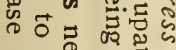

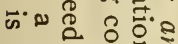

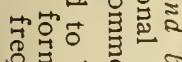

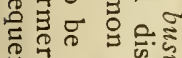

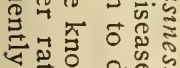

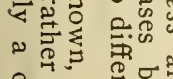

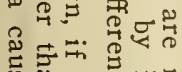

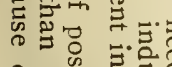

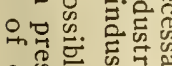
解. 政:-

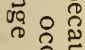

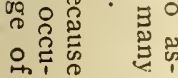

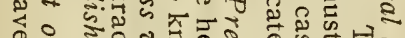

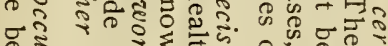

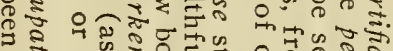

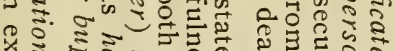

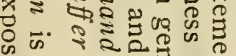

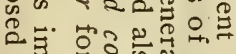

듕

एँ

लै०

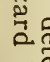

路高高

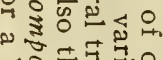

0.4
4

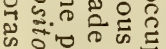

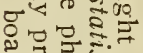

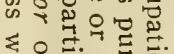

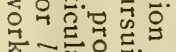

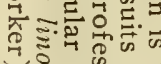

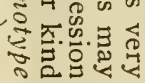

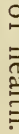

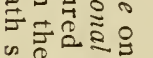

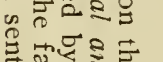
of

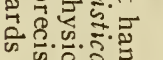

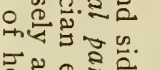

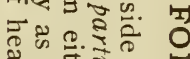

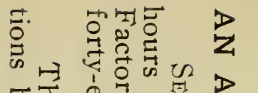

离

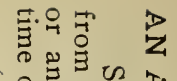

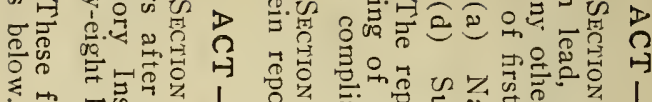

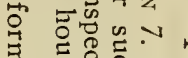

So

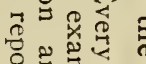

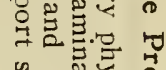

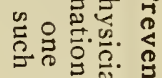

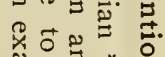

政: * *

的 *

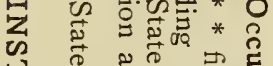

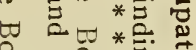

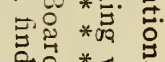

政:

品聂识**

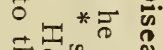

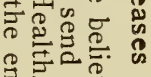

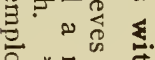

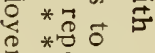

*ำ

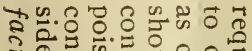

5.

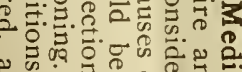

का

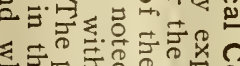

떠

전

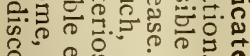

商

을

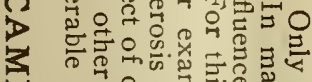

武

सx

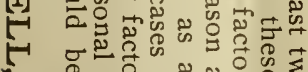

5 T

3 하의.

$\forall$ 送:

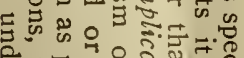

का

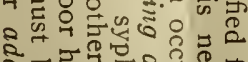

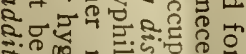

50.

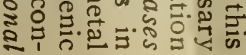

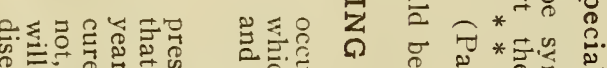

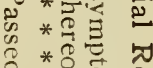

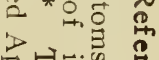

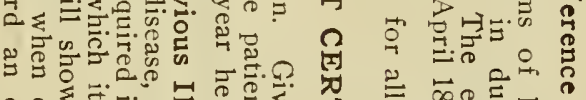

000

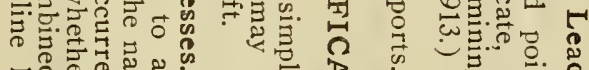

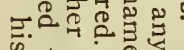

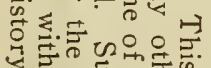

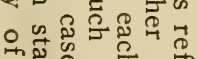

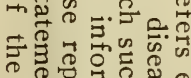

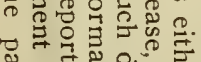

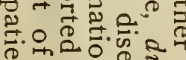

$\Rightarrow \rightarrow$ w

\&

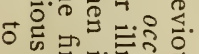

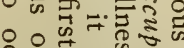

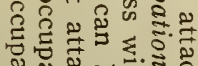

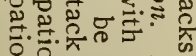

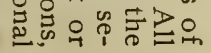

要

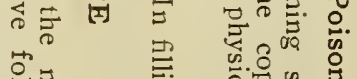

毫券

家。

章品

政:

喓言

응

政

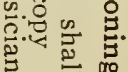

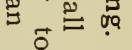

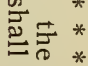

带此 *

(1)

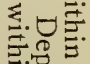

호을

\%

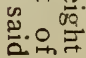

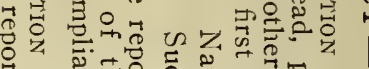

ॠ

公

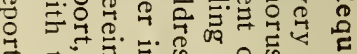

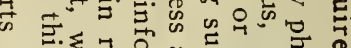

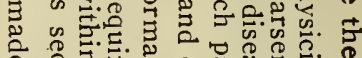

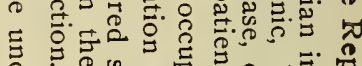

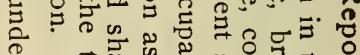

E.

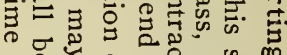

कर 0 <

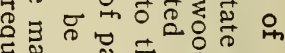

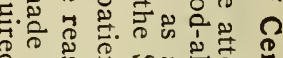

है

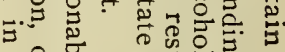

요원

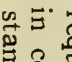

范。

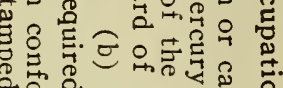

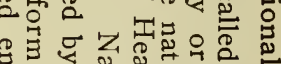

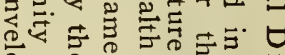

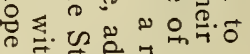

可产蛋

득 可

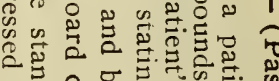

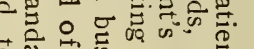

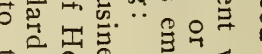

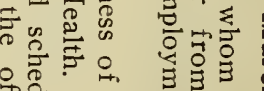

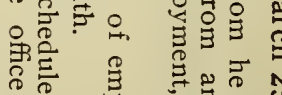

8

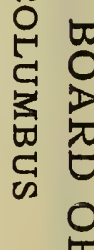

엄

杢

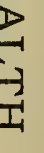

畒

约

$-1$

$\rightarrow$

स]

$\infty$
0
0
0
0
0
$I$
$I$

(1)

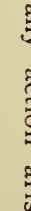

玤

"ลี

을

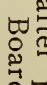

20

年

我到

离?

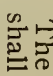

㤩导

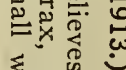

․․ㅇ

s.

क융 $\gg$

दु

เ 8 क⿻

象寻

ᄀ

용요

苟亩影

a.

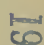

5

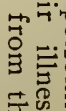

志思苛 


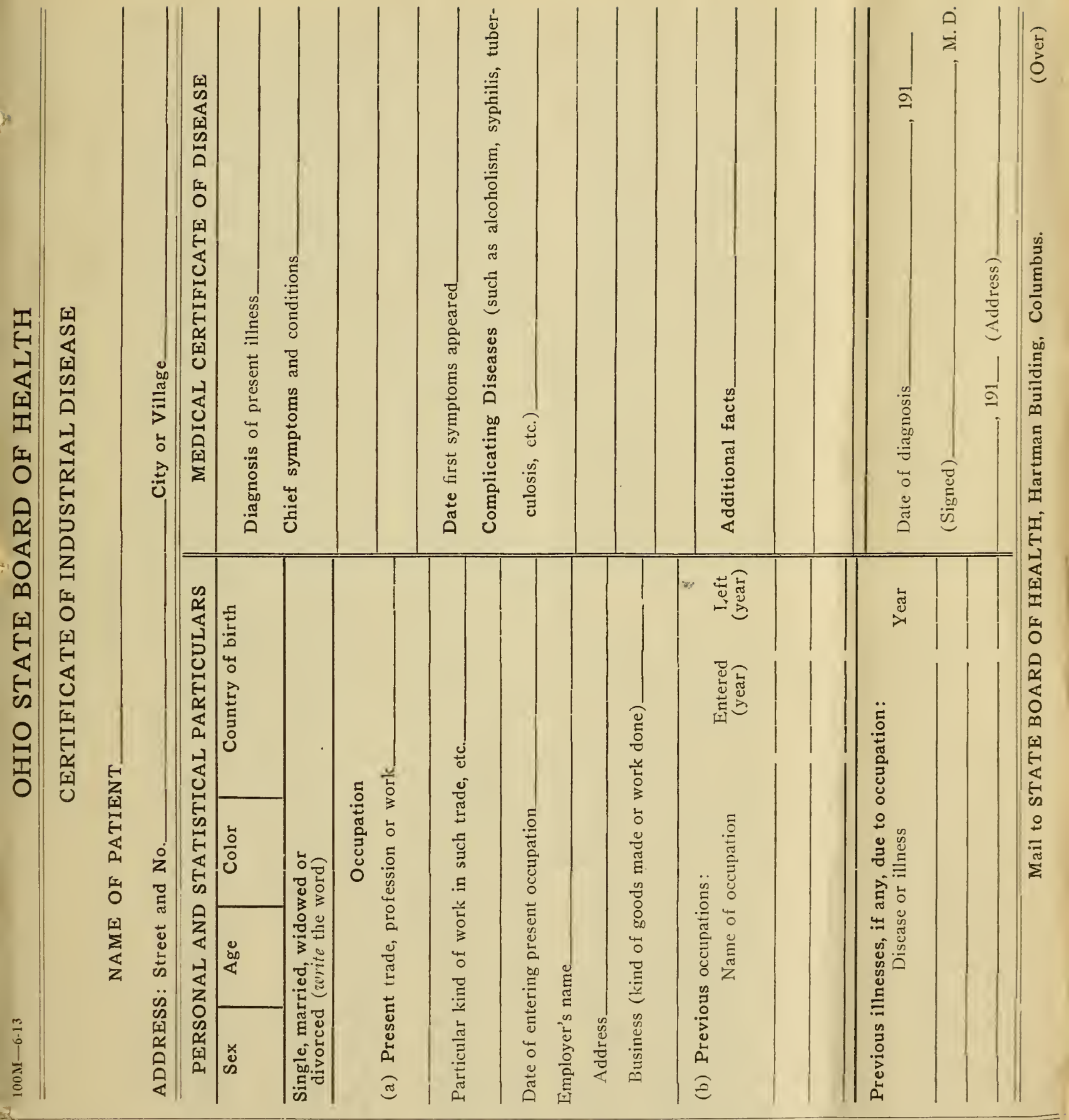

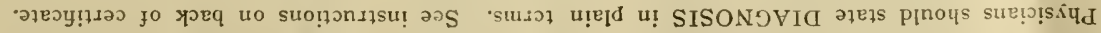

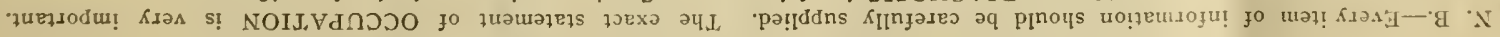




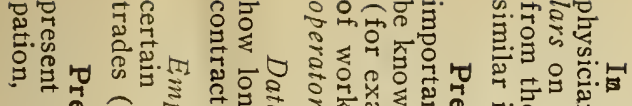

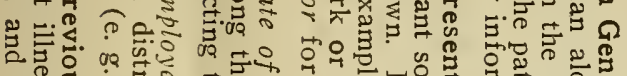

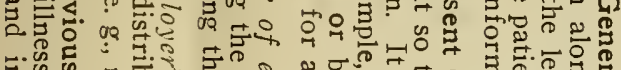

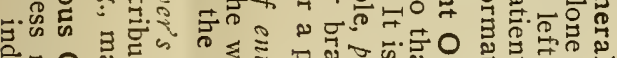

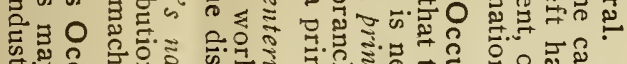

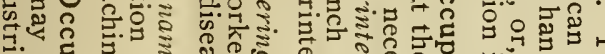

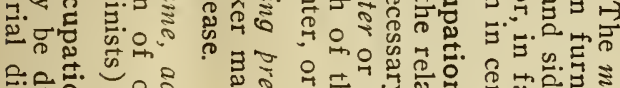

जिं

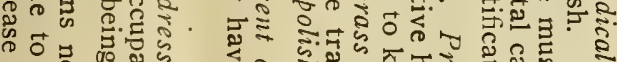

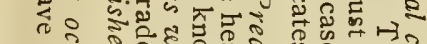

फ

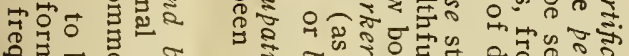

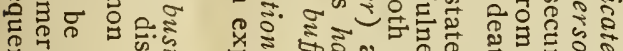

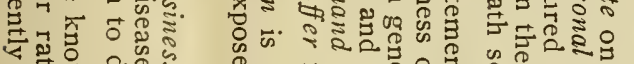

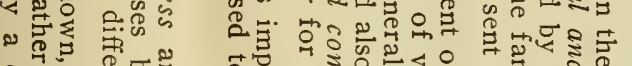

ङ

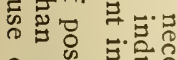

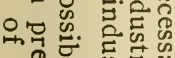

ล

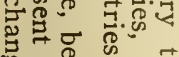

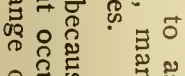

कि

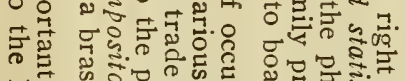

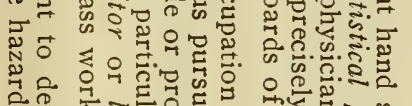

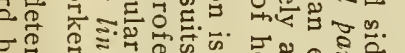

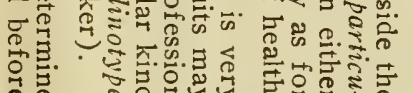

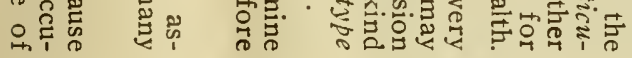

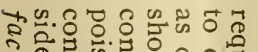

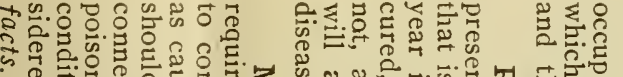

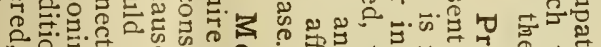

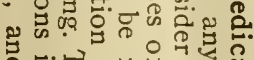

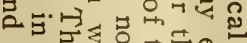

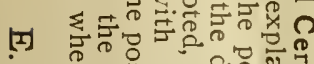

य

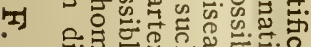

每

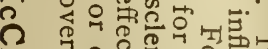

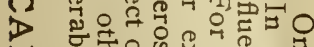

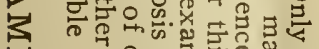

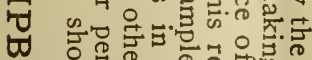

Ш

स

-

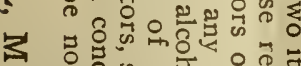

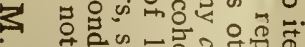

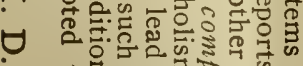

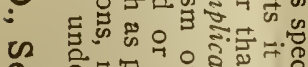

๙

กิ

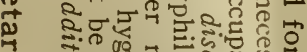

4 苛

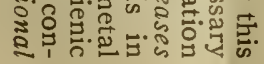

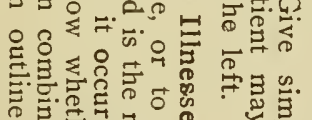

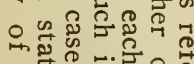

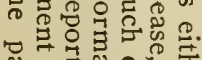

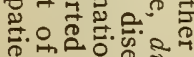

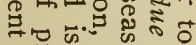

ผ

प율

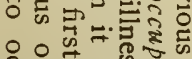

它昆

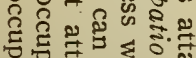

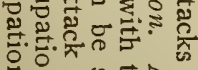

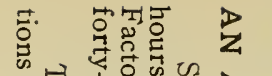

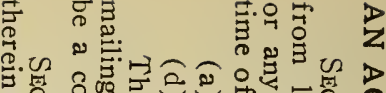

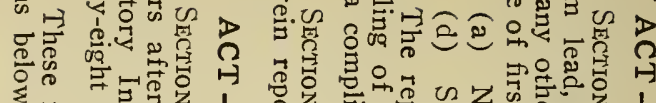

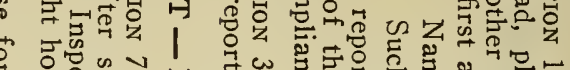

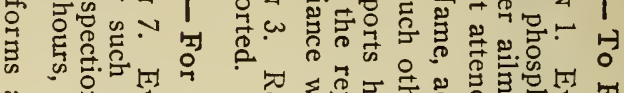

苛

声

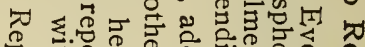

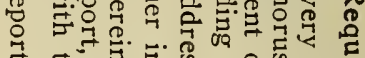

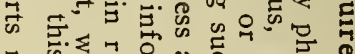

.

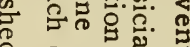

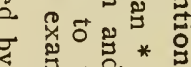

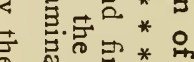

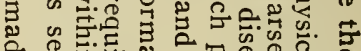

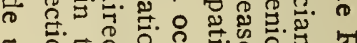

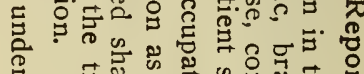

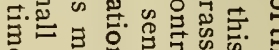

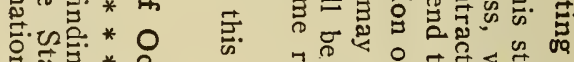

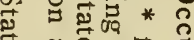

苛*实

एँ

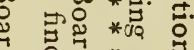

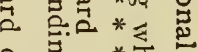

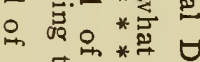

开 0 *穴

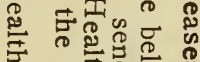

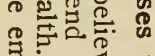

苟 总 क

क

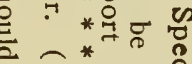

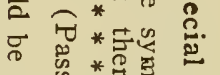

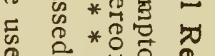

ฉ

क्ष

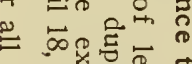

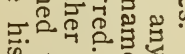

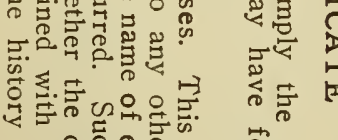

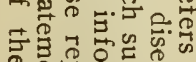

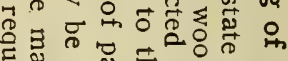

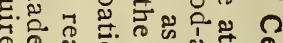

ล०

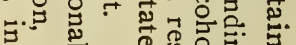

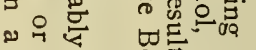

थ구

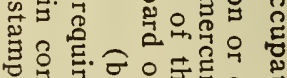

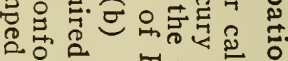

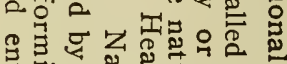

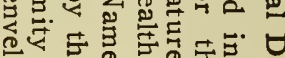

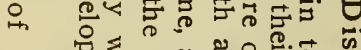

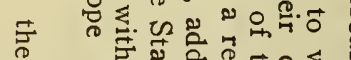

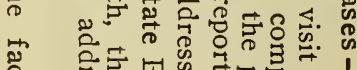

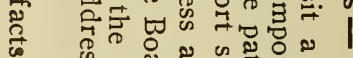

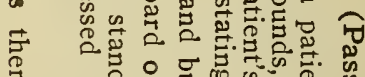

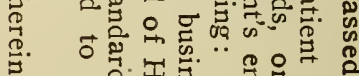

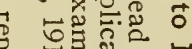

它项

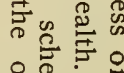

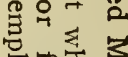

它

实

可

थै

II

II

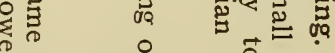

$\overrightarrow{0}^{*}$

总吉*

此*

$\overrightarrow{0} \leq$

造

它?

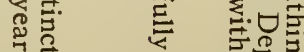

穴。

క.

急总

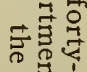

要

串.올

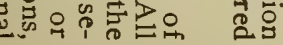

象官

串

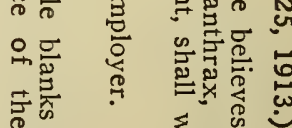

त्ञ

के

$\sum$ 品

ซึ?

ค.

온

证

ลี

象实

总宁 N

록웅

붕ㅁㅇ

울

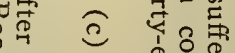

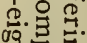

F

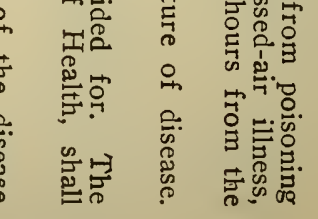

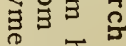




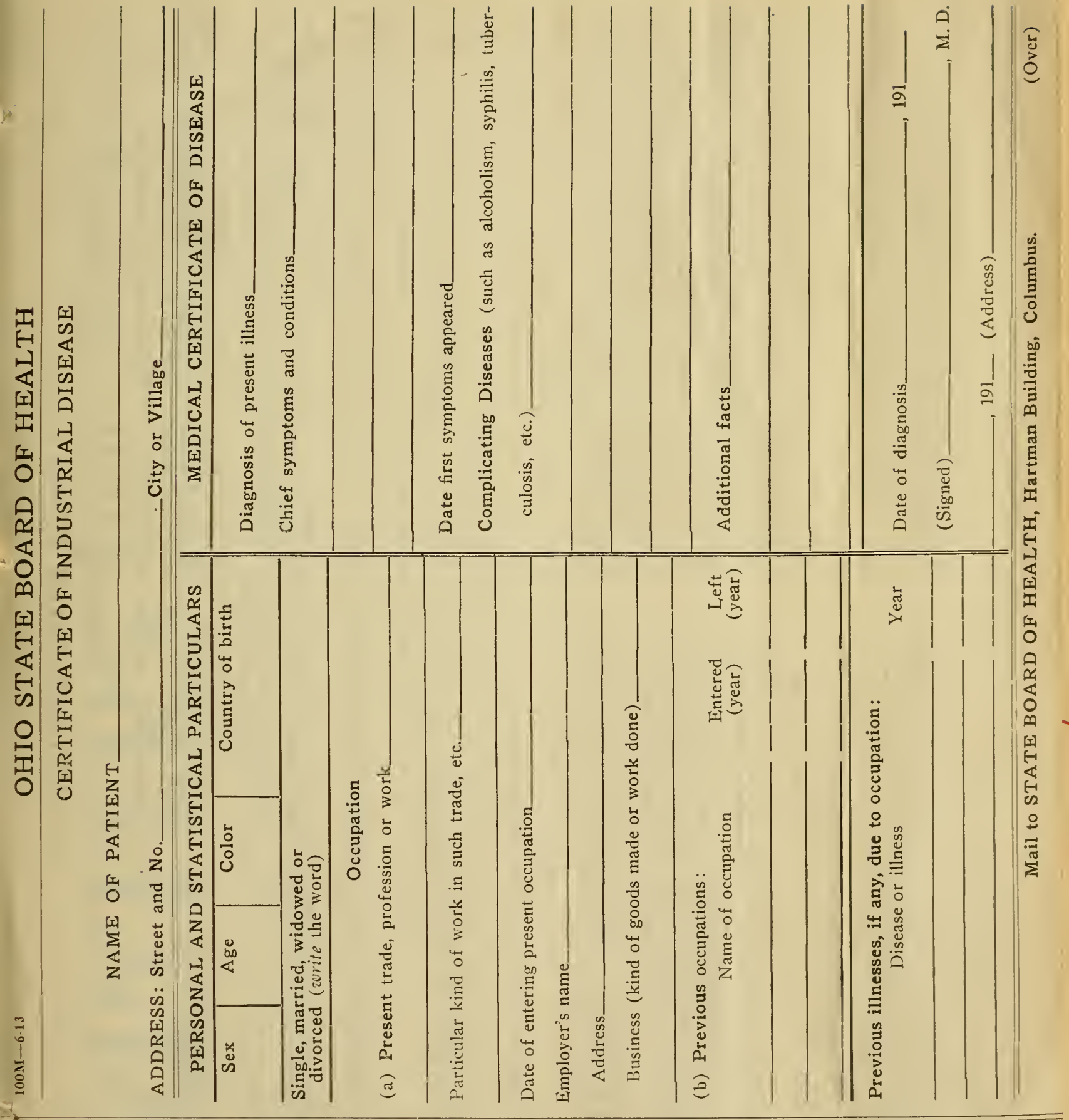

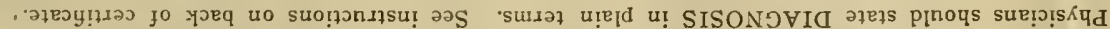

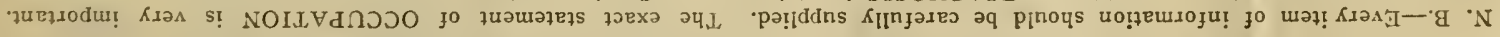

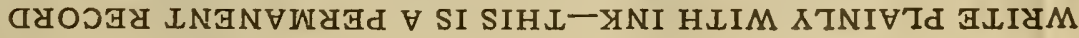




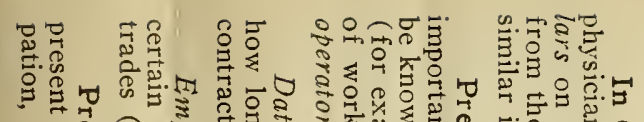

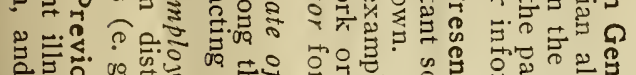

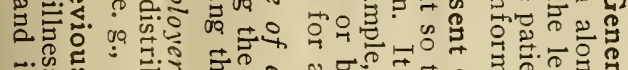

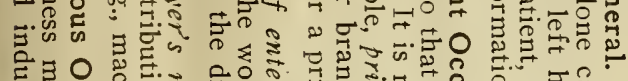

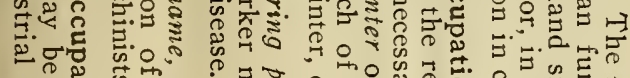

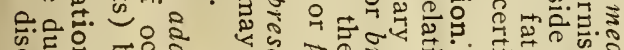
कृ

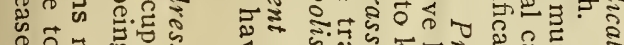

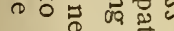

क'

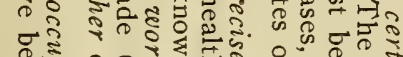

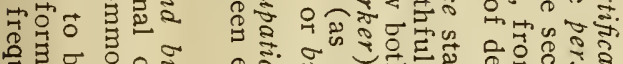

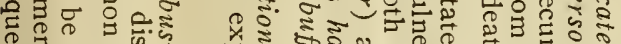

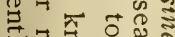

究点官 0 心

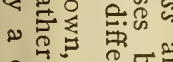

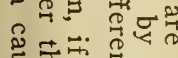

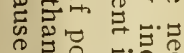

品点邑

응.

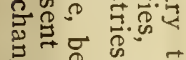

ำำ.

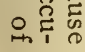

\section{I.)}

2.

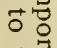

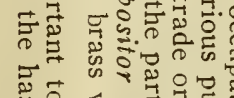

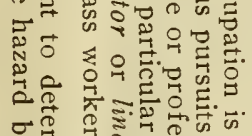

的政

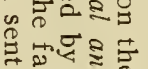

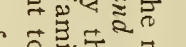

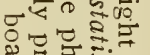

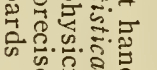

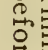

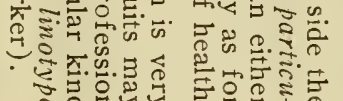

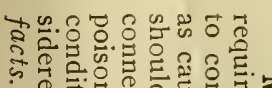

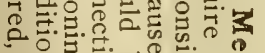

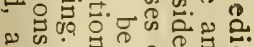

落.

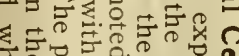

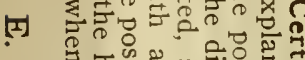

y

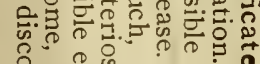

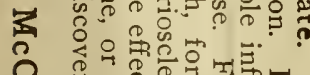

々

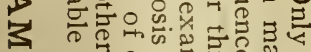

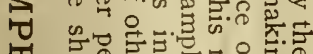

๒

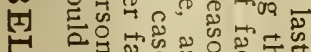

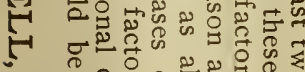

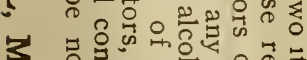

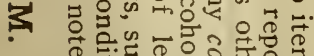

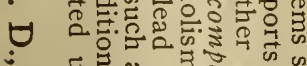

थ

ค

तै

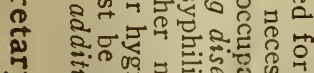

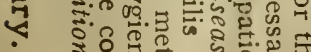

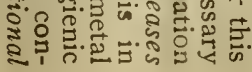

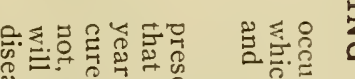

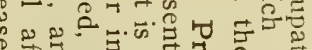

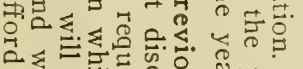

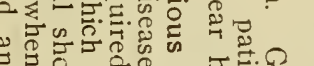

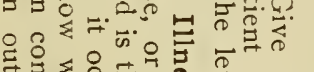

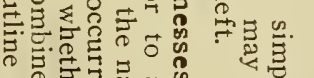

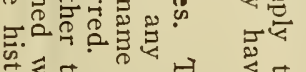

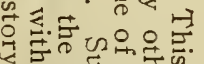

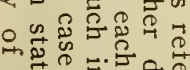

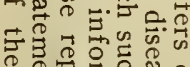

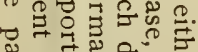

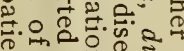

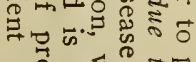

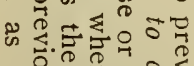
ธ

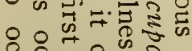
官总

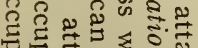
ฉ。 㝞嵒 容这.

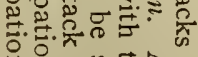
总点。只宫已。

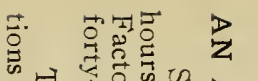

氞

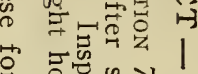

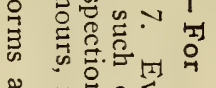

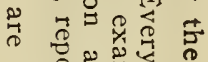

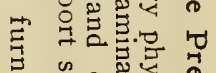

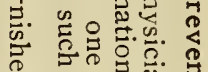

ฉ \&

प्ष

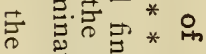

的的宫* *

2$$
\text { 苟寻它 }
$$$$
\text { 윽윰 }
$$$$
\text { ㄷ. 든 }
$$

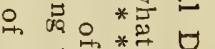$$
\text { 露 }
$$$$
\text { 政 }
$$

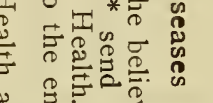

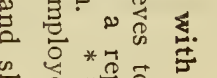$$
5 \text { क }
$$$$
\text { . } * 0 \text { 告 }
$$

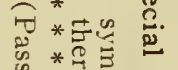

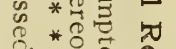$$
\text { 苦焉. }
$$$$
\text { 它 }
$$

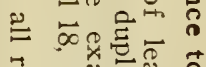

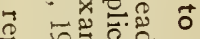$$
\text { 马 }
$$$$
\text { क }
$$

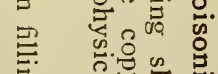$$
\text { :. }
$$$$
\exists \text { 鉎 }
$$

苞吉* *

象劳 *

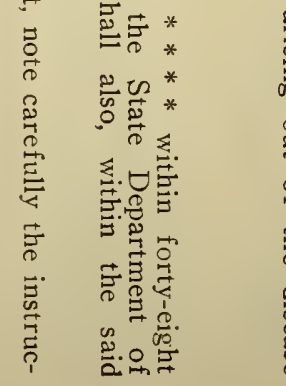

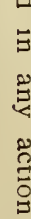$$
\text { Э }
$$

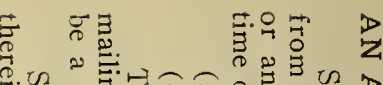

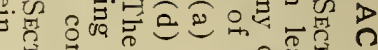

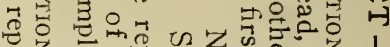

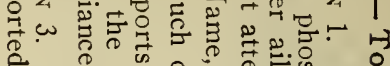

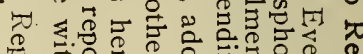

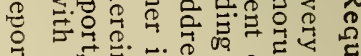

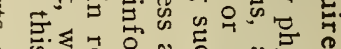

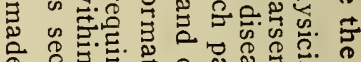

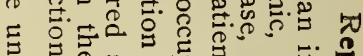

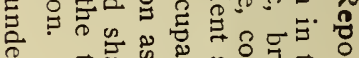
ㄹ. ०

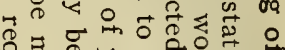

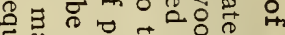

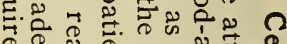

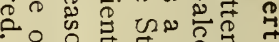

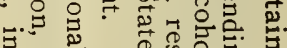

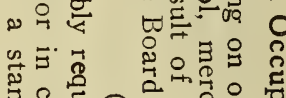

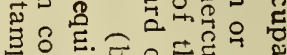

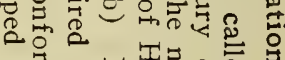

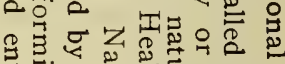

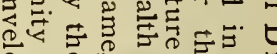

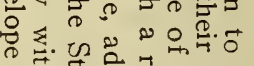

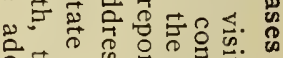

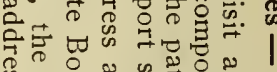

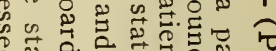

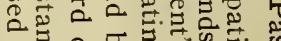

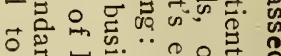

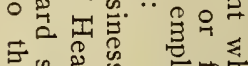

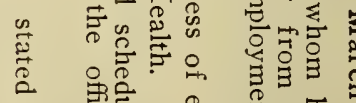

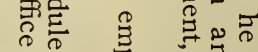

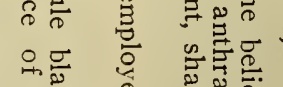
它芟

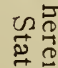
㱕。 召蛋 쿵ㅁㅇ 
Da

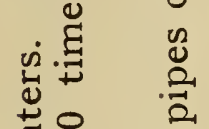

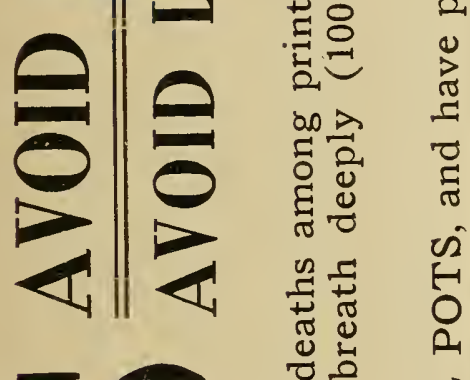

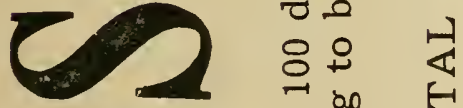
$\rightarrow$ bo

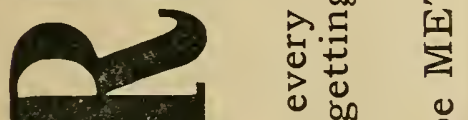
P 



\section{PRINTERS $\frac{\text { AVOID RONSUGAPTION }}{\text { AVOID LE }}$}

CONSUMPTION causes 29 out of every 100 deaths among printers. Stagnant air, iumes, gases, lead poisoning, lack of exercise, and forgetting to breath deeply (100 times each day) are the main reasons.

Hoods must be placed over linotype METAL POTS, and have pipes connecting, and leading out of doors.

Remember, pig-lead used in LINOTYPING is softer than lead of type. Handle it as little as possible. Then keep fingers away from mouth and lips. dust.

Drop pig-lead carefully into melting pot. SPLASHINGS of molten lead dry later and become lead

Do not shake crucible in order to blend MOLTEN LEAD better. It will blend of itself.

PLUNGERS on linotype machines should never be cleaned in the work room. Clean them in boxes in the open air. Avoid inhaling the dust.

GRAPHITE used for lubricating is not poisonous, but all dust is irritating to the lungs.

Avoid LEAD DUST, as much as possible, when trimming and mitreing, or when sawing.

Remove LEAD DUST from type cases in the open air, or by means of a vacuum cleaner.

Never put TYPE into the mouth, or moisten fingers to get hold of type.

Benzine and other cleaners occasionally contain DEADLY POISONS, which poison if gotten onto the skin or when inhaled.

Insist upon having GOOD VENTILATION in the office or factory, and insist that FLOORS SHOULD NOT BE SWEPT during working hours.

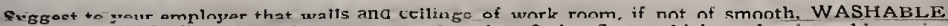
SURFACE, should be lime-washed once a year; that close-fitting floors which can be cleaned by moist methods are desirable; and that type-cases should fit closely on the floor, or have legs high enough to brush under.

Eat a good breakfast before beginning work. Food in the stomach, especially MILK, helps to prevent lead-poisoning.

Do not eat FOOD or use TOBACCO, while at work, until you have washed your hands, because of the danger of actually feeding lead to yourself. Do not use a "common" DRINKING CUP; such a cup may be employed by a tuberculous or otherwise infected person. Wash hands thoroughly with warm water and soap. Have your own towel and soap. Rinse the mouth and clean the fingernails before eating. Don't use fingernails for TOOTHPICKS.

Don't spit on the floor. Use CUSPIDORS and see that they are cleaned daily.

Eat your LUNCH outside of the work room.

Do not wear WORKING CLOTHES too long without change.

Hang STREET CLOTHES where they will not be exposed to the dust of the work room.

Gas and electric lights should be shaded to prevent a glare. The EYES should be EXAMINED from time to time by a competent physician. Avoid ruining your sight by giving early attention to eye-strain. Headaches, blurred vision, red and inflamed eyes, dancing spots before the eyes, twitching of the eye-lids, are some of the first signs of eye-strain.

Insufficient LIGHT may impair the general health.

BATHE frequently, and brush the TEETH each night.

Avoid ALCOHOL. It increases the danger of lead-poisoning.

Have a good BOWEL MOVEMENT each day.

EXERCISE in the fresh air as much as possible.

BE EXAMINED BY A DOCTOR occasionally to protect yourself against the effects of your trade.

ISSUED JANUARY, 1916

Division of Industrial Hygiene Ohio State Board of Health

E. F. MCCAMPBELL. Ph.D.. M.o. Secretary and Exocultivo officer COLUMBUS, OHIO 

Instructions to Employes in Dusty Trades

\section{DANGERS OF DUST}

1. Don't breathe dust of any kind-it causes colds, consumption and pneumonia.

2. Don't sweep during working hours-it spreads germs of all kinds.

3. Don't work in dusty air. Stop the dust or wear a dust protector over your mouth and nose.

4. Dust breathed into your lungs is never breathed out again.

5. If you breathe dust you are bound to cough.

6. Coughing or spitting is nature's warning that your lungs are in danger.

7. If you hem or cough every day see a doctor at once.

OHIO STATE BOARD OE HEALTH E. F. McCampbell, Ph.D.. M.D.

Secretary and Executive Officer 



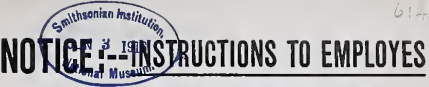

\section{HOW TO PREVENT LEAD POISONING}

(1) All workers exposed to lead dusts, lead fumes, lead solutions and lead compounds are liable to poisoning. These poisons get into the body through the nose while breathing, or through the mouth when chewing, or swallowing, or wetting the lips.

(2) Do all you can to keep down dust. When sweeping or cleaning, always dampen with water, oil or wet sawdust. Where dust can not be kept down, you must wear a respirator. This must be cleaned out at least once a day.

(3) Eat breakfast before going to work. Drink milk at meals, and if possible once between meals. Do not eat meals in workroom. Leave workroom at meal times.

(4) Keep dirty fingers out of your mouth, and off of your food, and whatever goes into your mouth. Wash hands, arms and face with warm water and soap before eating, going to the toilet, or quitting the workroom. Clean your lips and rinse out your mouth before eating or drinking.

(5) A mustache, if worn, must be kept short. Do not wear a beard. Keep fingernails clean and cut short, also loose skin about the nails or hands.

(6) Do not chew tobacco or gam while at work. Avoid the use of intoxicants in any form, as they promote lead poisoning.

(7) Take a full bath with warm water and soap at least twice a week.

(8) You must wear overalls and jumpers while at work. Wear a cap if exposed to dust or fumes. Do not wear your working clothes outside of the working place.

(9) Keep your bowels moving if possible once a day. Report to your foreman if you notice (1) loss of appetite, (2) poor sleep, (3) indigestion, (4) continual constipation, (5) vomiting, (6) pains in stomach, (7) dizziness, $(8)$ continual headache, or (9) weakness in arms, limbs or body.

NOTE: Lead poisoning brings on Paralysis of the wrists and arms, hardens the arteries, causes chronic diseases, and hastens old age and death. WORKMAN, PROTECT YOURSELF. Your employer and the Board of Health cannot do all for you. OBSERVE THE ABOVE PRECAUTIONS.

\section{OHIO STATE BOARD OF HEALTH,}

E. F. McCAMPBELL, Ph.D., M.D.,

Columbus, Ohio.

Secretary and Executive Officer. 

Lead does not get into the body through the skin. It gets in through the nose or mouth. Hence it should be easy to avoid lead poisoning.

Furthemore, 19 out of every 100 deaths among painters are due to CONSUMPTION. Twice as many painters die of consumption as carpenters. Undoubtedly working with poisons causes the difference. Poisons predispose to consumption.

Eat a good breakfast before beginning work. A FULL STOMACH lessens the danger of lead poisoning.

MILK is the best antidote for lead. Drink it at lunch or during the day.

Do not put food or tobacco into your mouth with dirty FINGERS. In other wordș, do not feed lead to yourself.

RINSE OFF LIPS before eating or drinking. Keep mustache short so as not to touch your food or drink. A mustache is a danger since it collects dust.

Wash HANDS thoroughly with warm water and soap before eating whenever you can. Otherwise hold sandwiches, pie, etc., between clean pieces of paper.

Eat your LUNCH outside of the room or place where painting or sanding is done.

Keep fingernails clean. Do not use them for TOOTHPICKS.

It is a good plan to rinse out your mouth before eating or drinking.

When sanding avoid breathing the DUST or allowing it to settle on your lips. Wear some sort of a respirator which will keep you from breathing the dust.

While SANDING do not chew tobacco or gum. The chewing movements always cause a little swallowing which YOU DO NOT NOTICE. Licking the lips. chewing and this "little swallowing" causes the most of lead poisoning.

Do not moisten your lips with your tongue. Each time you lick in some lead particles which have settled on your lips.

Prevent DUST as much as possible. Look out for dirty, dusty drop-cloths. Do not shake themm

When possible do sanding with a little MINERAL OIL present to absorb the dust. Such oil is cheap and can be used on almost any kind of work. lead.

Do not put tobacco in outside POCKETS where dust collects. Such dust usually contains some

Brush your TEETH at least in the morning and at night, the latter before going to bed.

Avoid ALCOHOLIC DRINKS - they make you more liable to lead poisoning.

Do not use a dirty cloth or rag to wipe off your face, nose or lips.

Have a good BOWEL MOVEMENT each day - best time is just after breakfast. Make the habit regular.

Hang street CLOTHES away from the paint and dust of the work place.

Have overalls and jumpers washed at least once a week.

Painters should get a good bath at least once a week, and use plenty of SOAP.

Remember also that all DRYERS and PAINT REMOVERS are very poisonous. Do not breathe their fumes or odors in a closed-up space. Have good ventilation.

TURPENTINE damages the kidneys sooner or later.

BE EXAMINED BY A DOCTOR OCCASIONALLY.

\section{SYMPTOMS:}

Have you ever had rheumatism, kidney, stomach, or heart trouble? Each may be due to lead.

Some SYMPTOMS of actual LEAD POISONING:-

Cramps in stomach or bowels

Constipation

Bluish lines on gums

Diarrhoea

Nausea

Foul taste in mouth

Severe headache

Loss of weight

Loss of strength
Pains in joints or back

Numbness of arms or fingers

Weakness of wrist or toe

Acting strangely

Tremors

Dizziness or dizzy spelis

Nervousness

Pallor

Wrist-drop or "palsy"
ISSUEO JANUARY, 1916

Division of Industrial Hygiene

OHIO STATE BOARD OF HEALTH

E. F. MCCAMPBELL, Ph.D.. M.D. Socrotary ind Execulive Ollicar COLUMBUS, OHIO 

II. AVOID DANGERS TO HEALTH - Know What They Are. Here are some of them and their limits:

1. The AIR in most buildings heated by stoves, furnaces, steam or hot water is stagnant, too hot and too dry - dryer than that of the Sahara Desert. Hence sore throats, colds, and many forms of sickness.

GOOD AIR:

CIRCULATES - and by so doing tones up the human system by striking the skin, thus improving the blood-flow, and removing the "envelope of heat" which is given off by the body and constantly surrounds it. Use electric fans or cloth-windows or window-boards or ventilators. A just perceptible "breath" is enough (or air blowing 5 to 10 feet per second).

Has RELATIVE HUMIDITY of 45 to $65 \%$. Learn the use of the wetbulb thermometer. When not too cold, proper moisture can be had by admitting outdoor air (note cloth-windows). It may take 10 to 20 gallons of water per day for a six-room house. Few, if any, pan and water schemes will evaporate this amount of water.

Has a TEMPERATURE (indoors) of from 60 to $68^{\circ} \mathrm{F}$. This temperature feels perfectly comfortable when the air is properly moistened-it also saves on the coal bill.

Has no irritating FLOATING PARTICLES - dust, smoke, etc.

Has no noticeable GASES, FUMES OR VAPORS - these are especially dangers of modern industries. Any ODOR should be pleasant - the air should be just like that supplied by Nature.

Not POLLUTED by disease germs. Most so-called "air-borne diseases" are really caught by contact with, or close approach to, persons carrying the germs. Such "carriers" may not themselves be sick and also may be in total ignorance of their own menace. Avoid touching persons as much as possible. Keep beyond the breathing range, and, finally, wash your hands often to prevent carrying disease to your face and mouth.

\section{GOOD ILLUMINATION means:}

STEADY LIGHT from over the shoulders - 5-10 foot-candles of ARTIFICIAL light for ordinary eye work, $5 \mathrm{f}$. c. in foundries and similar p!aces, and $3 \mathrm{f}$. c. on the floors of general workrooms. Daylight COLOR is the best. DAYLIGHT should be three times stronger than artificial light. (A foot-candle is the amount of light given by a standard candle one foot away).

Absence of sharp contrasts of lights and shadows; have light-colored walls and ceilings to reflect light instead of absorbing it; unobstructed light, as from the upper half of windows - dirty windows may obstruct 4/5 of the light, while dark shades should not be used in workrooms, offices, or schools; protection of the eyes against brilliant and hot lights.

GOOD EYE-SIGHT - perhaps, after all, y.our eye-sight is defective and your eyes need examining - most headaches are from eyestrain - eyestrain may be as fatiguing, also, as the most arduous work.

3. POISONS. Workers with poisons should be properly instructed in their use. The COURTS have decided that the responsibility of such instruction rests upon the employer. In Ohio the State Department of Health stands prepared to issue INSTRUCTIONS for the avoidance of poisoning of all sorts. Most poisonings occur because employers and workers both CONSIDER the substances dealt with as too innocent to cause much harm. This attitude has unfortunately caused an immense amount of sickness, numerous deaths, and many LAW SUITS for large amounts of money. The State Laboratories will analyze suspected poisons used in connection with work, provided the sources, uses and reasons for the analyses are stated. The Department of Health will also supply INFORMATION as to the maximum amount of poisonous substances vrhich can be present in the air or may be taken into the system without causing symptoms - This insofar as it has information or can secure the same. By law, all instances of poisoning in connection with work must be brought TO THE NOTICE of the State Department of Health, which Department furnishes the proper blanks for recording the information and investigates the occurrence with a view to finding out how to avoid similar mishaps. Such reports cannot be used as evidence of the facts therein in any action arising out of the disease 
therein reported. Most poisonings occur through workers poisoning themselves, which is due to IGNORANCE or DISREGARD of instructions, but upon the employer rests the responsibility of supervision. The more COMMON POISONS used in the State of Ohio are about in order of their frequency of use and their liability to produce occupational disease: lead, benzine and benzol (naphtha, petrol, gasoline, etc.), turpentine and similar dryers, brass or zinc in the form of fumes; acids, alkalis, wood alcohol, anilin oil, carbon bisulphide, antimony, illuminating and fuel gas, sulphurated hydrogen, arsenic, phosphorus and mercury.

4. FATIGUE. A tired feeling is nature's warning to rest. Getting tired depends largely upon who you are and how well you may be. Work should promote health and a sense of well-being. "So tired" should never be heard at the end of a day's work, as it signifies exhaustion. All straining or heavy or rapid work should be tempered or subjected to changes so that no feeling of exhaustion results. This applies to the eyes, or to the hands, as well as to the whole body. True "Scientific Management" observes these principles and makes use of several methods of doing routine work. On the other hand, TOO LITTLE EXERCISE is a serious danger to large numbers of persons who are not actively employed. Variation, which will allow exercise, is the secret of efficiency and steadfastness. The best standard for fatigue or inactivity is that "tired feeling". Avoid.it.

5. TEMPERATURE. The exposure to heat produces thermic fever if the temperature goes beyond that of the human body $\left(98.6^{\circ}\right)$; beyond this, prostrations, muscular cramps and anemia are produced. Premature old age is a common result. When COMBINED WITH MOISTURE the effects upon the health are very much worse. For the best work, neither the wet nor dry thermometer should exceed $70^{\circ} \mathrm{F}$. The shower-bath is a most excellent way to control the circulation of the blood upon quitting the hot workplace preparatory to going outside. In almost all trades heat can be controlled or kept away from workmen. EXPOSURE TO COLD, pure and simple, is not dangerous to health provided enough clothing is worn and enough active work is performed. Cooling one part of the body while heating another is bad.

6. DIRT AND DISORDERED SURROUNDINGS are dangerous to health because they favor disease, obstruct light, make one less inclined to do a high class of work; also to observe health standards, correct habits and good morals, whether at home, at work, or elsewhere.

7. THE NATURAL DESIRES create great risks to health -

1. THIRST must be supplied with safe drinking water.

2. HUNGER must be supplied with clean, nourishing food eaten in a place free from poisons.

3. TOILETS, URINALS, SEWERAGE and GARBAGE devices must not engender disease.

4. SLEEP must not be disturbed and must last eight hours every night.

5. RECREATION must not be dissipative but recreative.

6. The desire for STIMULANTS is always abnormal to the healthy person; when present it is usually due to the effects of some of the dangers above cited or to a habit acquired through ignorance or bravado. Stimulants include alcohol, coffee, tea and certain drugs.

7. Make the day the routine of life - don't try to catch up on Sundays only.

IV. THE LAST FACTOR in the cure of this national health decline is that of providing for the cases of sickness which occur. It is THE RELIEF SCHEME. Just as we have accident insurance in order to compensate for accidents we should have sickness insurance in order to compensate for sickness. It is best to call this "HEALTH INSURANCE" for we wish to maintain health. Such insurance is the real active agent in bringing persons to observe the Laws of Health, to have Physical Examinations made and to see that their Surroundings are Healthful. To bring this about the insurance should be supported by the worker $(2 / 5)$, by the employer $(2 / 5)$, and by the state $(1 / 5)$. By this means every one is financially interested in prolonging health, increasing efficiency and maintaining a high standard of production.

WORK SHOULD PROMOTE HEALTH, NOT DESTROY IT 
III. AVOID DANGERS TO HEALTH - Know What They Are. Here are some of them and their limits:

1. The AIR in most buildings heated by stoves, furnaces, steam or hot water is stagnant, too hot and too dry - dryer than that of the Sahara Desert. Hence sore throats, colds, and many forms of sickness.

\section{GOOD AIR:}

CIRCULATES - and by so doing tones up the human system by striking the skin, thus improving the blood-flow, and removing the "envelope of heat" which is given off by the body and constantly surrounds it. Use electric fans or cloth-windows or window-boards or ventilators. A just perceptible "breath" is enough (or air blowing 5 to 10 feet per second).

Has RELATIVE HUMIDITY of 45 to $65 \%$. Learn the use of the wetbulb thermometer. When not too cold, proper moisture can be had by admitting outdoor air (note cloth-windows). It may take 10 to 20 gallons of water per day for a six-room house. Few, if any, pan and water schemes will evaporate this amount of water.

Has a TEMPERATURE (indoors) of from 60 to $68^{\circ} \mathrm{F}$. This temperature feels perfectly comfortable when the air is properly moistened - it also saves on the coal bill.

Has no irritating FLOATING PARTICLES - dust, smoke, etc.

Has no noticeable GASES, FUMES OR VAPORS - these are especially dangers of modern industries. Any ODOR should be pleasant - the air should be just like that supplied by Nature.

Not POLLUTED by disease germs. Most so-called "air-borne diseases" are really caught by contact with, or close approach to, persons carrying the germs. Such "carriers" may not themselves be sick and also may be in total ignorance of their own menace. Avoid touching persons as much as possible. Keep beyond the breathing range, and, finally, wash your hands often to prevent carrying disease to your face and mouth.

\section{GOOD ILLUMINATION means:}

STEADY LIGHT from over the shoulders - 5-10 foot-candles of ARTIFICIAL light for ordinary eye work, $5 \mathrm{f}$. c. in foundries and similar places, and $3 \mathrm{f}$. c. on the floors of general workrooms. Daylight COLOR is the best. DAYLIGHT should be three times stronger than artificial light. (A foot-candle is the amount of light given by a standard candle one foot away).

Absence of sharp contrasts of lights and shadows; have light-colored walls and ceilings to reflect light instead of absorbing it; unobstructed light, as from the upper half of windows - dirty windows may obstruct $4 / 5$ of the light, while dark shades should not be used in workrooms, offices, or schools; protection of the eyes against brilliant and hot lights.

GOOD EYE-SIGHT - perhaps, after all, your eye-sight is defective and your eyes need examining - most headaches are from eyestrain - eyestrain may be as fatiguing, also, as the most arduous work.

3. POISONS. Workers with poisons should be properly instructed in their use. The COURTS have decided that the responsibility of such instruction rests upon the employer. In Ohio the State Department of Health stands prepared to issue INSTRUCTIONS for the avoidance of poisoning of all sorts. Most poisonings occur because employers and workers both CONSIDER the substances dealt with as too innocent to cause much harm. This attitude has unfortunately caused an immense amount of sickness, numerous deaths, and many LAW SUITS for large amounts of money. The State Laboratories will analyze suspected poisons used in connection with work, provided the sources, uses and reasons for the analyses are stated. The Department of Health will also supply INFORMATION as to the maximum amount of poisonous substances which can be present in the air or may be taken into the system without causing symptoms - This insofar as it has information or can secure the same. By law, all instances of poisoning in connection with work must be brought TO THE NOTICE of the State Department of Health, which Department furnishes the proper blanks for recording the information and investigates the occurrence with a view to finding out how to avoid similar mishaps. Such reports cannot be used as evidence of the facts therein in any action arising out of the disease 
therein reported. Most poisonings occur through workers poisoning themselves, which is due to IGNORANCE or DISREGARD of instructions, but upon the employer rests the responsibility of supervision. The more COMMON POISONS used in the State of Ohio are about in order of their frequency of use and their liability to produce occupational disease: lead, benzine and benzol (naphtha, petrol, gasoline, etc.), turpentine and similar dryers, brass or zinc in the form of fumes; acids, alkalis, wood alcohol, anilin oil, carbon bisulphide, antimony, illuminating and fuel gas, sulphurated hydrogen, arsenic, phosphorus and mercury.

4. FATIGUE. A tired feeling is nature's warning to rest. Getting tired depends largely upon who you are and how well you may be. Work should promote health and a sense of well-being. "So tired" should never be heard at the end of a day's work, as it signifies exhaustion. All straining or heavy or rapid work should be tempered or subjected to changes so that no feeling of exhaustion results. This applies to the eyes, or to the hands, as well as to the whole body. True "Scientific Management" observes these principles and makes use of several methods of doing routine work. On the other hand, TOO LITTLE EXERCISE is a serious danger to large numbers of persons who are not actively employed. Variation, which will allow exercise, is the secret of efficiency and steadfastness. The best standard for fatigue or inactivity is that "tired feeling". Avoid it.

5. TEMPERATURE. The exposure to heat produces thermic fever if the temperature goes beyond that of the human body $\left(98.6^{\circ}\right)$; beyond this, prostrations, muscular cramps and anemia are produced. Premature old age is a common result. When COMBINED WITH MOISTURE the effects upon the health are very much worse. For the best work, neither the wet nor dry thermometer should exceed $70^{\circ} \mathrm{F}$. The shower-bath is a most excellent way to control the circulation of the blood upon quitting the hot workplace preparatory to going outside. In almost all trades heat can be controlled or kept away from workmen. EXPOSURE TO COLD, pure and simple, is not dangerous to health provided enough clothing is worn and enough active work is performed. Cooling one part of the body while heating another is bad.

6. DIRT AND DISORDERED SURROUNDINGS are dangerous to health because they favor disease, obstruct light, make one less inclined to do a high class of work; also to observe health standards, correct habits and good morals, whether at home, at work, or elsewhere.

7. THE NATURAL DESIRES create great risks to health -

1. THIRST must be supplied with safe drinking water.

2. HUNGER must be supplied with clean, nourishing food eaten in a place free from poisons.

3. TOILETS, URINALS, SEWERAGE and GARBAGE devices must not engender disease.

4. SLEEP must not be disturbed and must last eight hours every night.

5. RECREATION must not be dissipative but recreative.

6. The desire for STIMULANTS is always abnormal to the healthy person; when present it is usually due to the effects of some of the dangers above cited or to a habit acquired through ignorance or bravado. Stimulants include alcohol, coffee, tea and certain drugs.

7. Make the day the routine of life - don't try to catch up on Sundays only.

IV. THE LAST FACTOR in the cure of this national health decline is that of providing for the cases of sickness which occur. It is THE RELIEF SCHEME. Just as we have accident insurance in order to compensate for accidents we should have sickness insurance in order to compensate for sickness. It is best to call this "HEALTH INSURANCE" for we wish to maintain health. Such insurance is the real active agent in bringing persons to observe the Laws of Health, to have Physical Examinations made and to see that their Surroundings are Healthful. To bring this about the insurance should be supported by the worker $(2 / 5)$, by the employer $(2 / 5)$, and by the state $(1 / 5)$. By this means every one is financially interested in prolonging health, increasing efficiency and maintaining a high standard of production. 
III. AVOID DANGERS TO HEALTH - Know What They Are. Here are some of them and their limits:

1. The AIR in most buildings heated by stoves, furnaces, steam or hot water is stagnant, too hot and too dry - dryer than that of the Sahara Desert. Hence sore throats, colds, and many forms of sickness.

\section{GOOD AIR:}

CIRCULATES - and by so doing tones up the human system by striking the skin, thus improving the blood-flow, and removing the "envelope of heat" which is given off by the body and constantly surrounds it. Use electric fans or cloth-windows or window-boards or ventilators. A just perceptible "breath" is enough (or air blowing 5 to 10 feet per second).

Has RELATIVE HUMIDITY of 45 to $65 \%$. Learn the use of the rivetbulb thermometer. When not too cold, proper moisture can be had by admitting outdoor air (note cloth-windows). It may take 10 to 20 gallons of water per day for a six-room house. Few, if any, pan and water schemes will evaporate this amount of water.

Has a TEMPERATURE (indoors) of from 60 to $68^{\circ} \mathrm{F}$. This temperature feels perfectly comfortable when the air is properly moistened - it also saves on the coal bill.

Has no irritating FLOATING PARTICLES - dust, smoke, etc.

Has no noticeable GASES, FUMES OR VAPORS - these are especially dangers of modern industries. Any ODOR should be pleasant - the air should be just like that supplied by Nature.

Not POLLUTED by disease germs. Most so-called "air-borne diseases" are really caught by contact with, or close approach to, persons carrying the germs. Such "carriers" may not themselves be sick and also may be in total ignorance of their own menace. Avoid touching persons as much as possible. Keep beyond the breathing range, and, finally, wash your hands of ten to prevent carrying disease to your face and mouth.

\section{GOOD ILLUMINATION means:}

STEADY LIGHT from over the shoulders - 5-10 foot-candles of ARTIFICIAL light for ordinary eye work, $5 \mathrm{f}$. c. in foundries and similar p.aces. and $3 \mathrm{f}$. c. on the floors of general workrooms. Daylight COLOR is the best. DAYLIGHT should be three times stronger than artificial light. (A foot-candle is the amount of light given by a standard candle one foot away).

Absence of sharp contrasts of lights and shadows; have light-colored walls and ceilings to reflect light instead of absorbing it; unobstructed light, as from the upper half of windows - dirty windows may obstruct $4 / 5$ of the light, while dark shades should not be used in workrooms, offices, or schools; protection of the eyes against brilliant and hot lights.

GOOD EYE-SIGHT - perhaps, after all, your eye-sight is defective and your eyes need examining - most headaches are from eyestrain - eyestrain may be as fatiguing, also, as the most arduous work.

3. POISONS. Workers with poisons should be properly instructed in their use. The COURTS have decided that the responsibility of such instruction rests upon the employer. In Ohio the State Department of Health stands prepared to issue INSTRUCTIONS for the avoidance of poisoning of all sorts. Most poisonings occur because employers and workers both CONSIDER the substances dealt with as too innocent to cause much harm. This attitude has unfortunately caused an immense amount of sickness, numerous deaths, and many LAW SUITS for large amounts of money. The State Laboratories will analyze suspected poisons used in connection with work, provided the sources, uses and reasons for the analyses are stated. The Department of Health will also supply INFORMATION as to the maximum amount of poisonous substances which can be present in the air or may be taken into the system without causing symptoms - This insofar as it has information or can secure the same. By law, all instances of poisoning in connection with work must be brought TO THE NOTICE of the State Department of Health, which Department furnishes the proper blanks for recording the information and investigates the occurrence with a view to finding out how to avoid similar mishaps. Such reports cannot be used as evidence of the facts therein in any action arising out of the disease 
therein reported. Most poisonings occur through workers poisoning themselves, which is due to IGNORANCE or DISREGARD of instructions, but upon the employer rests the responsibility of supervision. The more COMMON POISONS used in the State of Ohio are about in order of their frequency of use and their liability to produce occupational disease: lead, benzine and benzol (naphtha, petrol, gasoline, etc.), turpentine and similar dryers, brass or zinc in the form of fumes; acids, alkalis, wood alcohol, anilin oil, carbon bisulphide, antimony, illuminating and fuel gas, sulphurated hydrogen, arsenic, phosphorus and mercury.

4. FATIGUE. A tired feeling is nature's warning to rest. Getting tired depends largely upon who you are and how well you may be. Work should promote health and a sense of well-being. "So tired" should never be heard at the end of a day's work, as it signifies exhaustion. All straining or heavy or rapid work should be tempered or subjected to changes so that no feeling of exhaustion results. This applies to the eyes, or to the hands, as well as to the whole body. True "Scientific Management" observes these principles and makes use of several methods of doing routine work. On the other hand, TOO LITTLE EXERCISE is a serious danger to large numbers of persons who are not actively employed. Variation, which will allow exercise, is the secret of efficiency and steadfastness. The best standard for fatigue or inactivity is that "tired feeling". Avoid it.

5. TEMPERATURE. The exposure to heat produces thermic fever if the temperature goes beyond that of the human body $\left(98.6^{\circ}\right)$; beyond this, prostrations, muscular cramps and anemia are produced. Premature old age is a common result. When COMBINED WITH MOISTURE the effects upon the health are very much worse. For the best work, neither the wet nor dry thermometer should exceed $70^{\circ} \mathrm{F}$. The shower-bath is a most excellent way to control the circulation of the blood upon quitting the hot workplace preparatory to going outside. In almost all trades heat can be controlled or kept away from workmen. EXPOSURE TO COLD, pure and simple, is not dangerous to health provided enough clothing is worn and enough active work is performed. Cooling one part of the body while heating another is bad.

6. DIRT AND DISORDERED SURROUNDINGS are dangerous to health because they favor disease, obstruct light, make one less-inclined to do a high class of work; also to observe health standards, correct habits and good morals, whether at home, at work, or elsewhere.

7. THE NATURAL DESIRES create great risks to health -

1. THIRST must be supplied with safe drinking water.

2. HUNGER must be supplied with clean, nourishing food eaten in a place free from poisons.

3. TOILETS, URINALS, SEWERAGE and GARBAGE devices must not engender disease.

4. SLEEP must not be disturbed and must last eight hours every night.

5. RECREATION must not be dissipative but recreative.

6. The desire for STIMULANTS is always abnormal to the healthy person; when present it is usually due to the effects of some of the dangers above cited or to a habit acquired through ignorance or bravado. Stimulants include alcohol, coffee, tea and certain drugs.

7. Make the day the routine of life-don't try to catch up on Sundays only.

IV. THE LAST FACTOR in the cure of this national health decline is that of providing for the cases of sickness which occur. It is THE RELIEF SCHEME. Just as we have accident insurance in order to compensate for accidents we should have sickness insurance in order to compensate for sickness. It is best to call this "HEALTH INSURANCE" for we wish to maintain health. Such insurance is the real active agent in bringing persons to observe the Laws of Health, to have Physical Examinations made and to see that their Surroundings are Healthful. To bring this about the insurance should be supported by the worker $(2 / 5)$, by the employer $(2 / 5)$, and by the state $(1 / 5)$. By this means every one is financially interested in prolonging health, increasing efficiency and maintaining a high standard of production. 
III. AVOID DANGERS TO HEALTH - Know What They Are. Here are some of them and their limits:

1. The AIR in most buildings heated by stoves, furnaces, steam or hot water is stagnant, too hot and too dry - dryer than that of the Sahara Desert. Hence sore throats, colds, and many forms of sickness.

\section{GOOD AIR:}

CIRCULATES - and by so doing tones up the human system by striking the skin, thus improving the blood-flow, and removing the "envelope of heat" which is given off by the body and constantly surrounds it. Use electric fans or cloth-windows or window-boards or ventilators. A just perceptible "breath" is enough (or air blowing 5 to 10 feet per second).

Has RELATIVE HUMIDITY of 45 to $65 \%$. Learn the use of the wetbulb thermometer. When not too cold, proper moisture can be had by admitting outdoor air (note cloth-windows). It may take 10 to 20 gallons of water per day for a six-room house. Few, if any, pan and water schemes will evaporate this amount of water.

Has a TEMPERATURE (indoors) of from 60 to $68^{\circ} \mathrm{F}$. This temperature feels perfectly comfortable when the air is properly moistened - it also saves on the coal bill.

Has no irritating FLOATING PARTICLES - dust, smoke, etc.

Has no noticeable GASES, FUMES OR VAPORS - these are especially dangers of modern industries. Any ODOR should be pleasant - the air should be just like that supplied by Nature.

Not POLLUTED by disease germs. Most so-called "air-borne diseases" are really caught by contact with, or close approach to, persons carrying the germs. Such "carriers" may not themselves be sick and also may be in total ignorance of their own menace. Avoid touching persons as much as possible. Keep beyond the breathing range, and, finally, wash your hands of ten to prevent carrying disease to your face and mouth.

\section{GOOD ILLUMINATION means:}

STEADY LIGHT from over the shoulders - 5-10 foot-candles of ARTIFICIAL light for ordinary eye work, $5 \mathrm{f}$. c. in foundries and similar places, and $f$. c. on the floors of general workrooms. Daylight COLOR is the best. DAYLIGHT should be three times stronger than artificial light. (A foot-candle is the amount of light given by a standard candle one foot away).

Absence of sharp contrasts of lights and shadows; have light-colored walls and ceilings to reflect light instead of absorbing it; unobstructed light, as from the upper half of windows - dirty windows may obstruct $4 / 5$ of the light, while dark shades should not be used in workrooms, offices, or schools; protection of the eyes against brilliant and hot lights.

GOOD EYE-SIGHT - perhaps, after all, your eye-sight is defective and your eyes need examining - most headaches are from eyestrain - eyestrain may be as fatiguing, also, as the most arduous work.

3. POISONS. Workers with poisons should be properly instructed in their use. The COURTS have decided that the responsibility of such instruction rests upon the employer. In Ohio the State Department of Health stands prepared to issue INSTRUCTIONS for the avoidance of poisoning of all sorts. Most poisonings occur because employers and workers both CONSIDER the substances dealt with as too innocent to cause much harm. This attitude has unfortunately caused an immense amount of sickness, numerous deaths, and many LAW SUITS for large amounts of money. The State Laboratories will analyze suspected poisons used in connection with work, provided the sources, uses and reasons for the analyses are stated. The Department of Health will also supply INFORMATION as to the maximum amount of poisonous substances which can be present in the air or may be taken into the system without causing symptoms - This insofar as it has information or can secure the same. By law, all instances of poisoning in connection with work must be brought TO THE NOTICE of the State Department of Health, which Department furnishes the proper blanks for recording the information and investigates the occurrence with a view to finding out how to avoid similar mishaps. Such reports cannot be used as evidence of the facts therein in any action arising out of the disease 
therein reported. Most poisonings occur through workers poisoning themselves, which is due to IGNORANCE or DISREGARD of instructions, but upon the employer rests the responsibility of supervision. The more COMMON POISONS used in the State of Ohio are about in order of their frequency of use and their liability to produce occupational disease: lead, benzine and benzol (naphtha, petrol, gasoline, etc.), turpentine and similar dryers, brass or zinc in the form of fumes; acids, alkalis, wood alcohol, anilin oil, carbon bisulphide, antimony, illuminating and fuel gas, sulphurated hydrogen, arsenic, phosphorus and mercury.

4. FATIGUE. A tired feeling is nature's warning to rest. Getting tired depends largely upon who you are and how well you may be. Work should promote health and a sense of well-being. "So tired" should never be heard at the end of a day's work, as it signifies exhaustion. All straining or heavy or rapid work should be tempered or subjected to changes so that no feeling of exhaustion results. This applies to the eyes, or to the hands, as well as to the whole body. True "Scientific Management" observes these principles and makes use of several methods of doing routine work. On the other hand, TOO LITTLE EXERCISE is a serious danger to large numbers of persons who are not actively employed. Variation, which will allow exercise, is the secret of efficiency and steadfastness. The best standard for fatigue or inactivity is that "tired feeling". Avoid it.

5. TEMPERATURE. The exposure to heat produces thermic fever if the temperature goes beyond that of the human body $\left(98.6^{\circ}\right)$; beyond this, prostrations, muscular cramps and anemia are produced. Premature old age is a common result. When COMBINED WITH MOISTURE the effects upon the health are very much worse. For the best work, neither the wet nor dry thermometer should exceed $70^{\circ} \mathrm{F}$. The shower-bath is a most excellent way to control the circulation of the blood upon quitting the hot workplace preparatory to going outside. In almost all trades heat can be controlled or kept away from workmen. EXPOSURE TO COLD, pure and simple, is not dangerous to health provided enough clothing is worn and enough active work is performed. Cooling one part of the body while heating another is bad.

6. DIRT AND DISORDERED SURROUNDINGS are dangerous to health because they favor disease, obstruct light, make one less inclined to do a high class of work; also to observe health standards, correct habits and good morals, whether at home, at work, or elsewhere.

7. THE NATURAL DESIRES create great risks to health -

1. THIRST must be supplied with safe drinking water.

2. HUNGER must be supplied with clean, nourishing food eaten in a place free from poisons.

3. TOILETS, URINALS, SEWERAGE and GARBAGE devices must not engender disease.

4. SLEEP must not be disturbed and must last eight hours every night.

5. RECREATION must not be dissipative but recreative.

6. The desire for STIMULANTS is always abnormal to the healthy person; when present it is usually due to the effects of some of the dangers above cited or to a habit acquired through ignorance or bravado. Stimulants include alcohol, coffee, tea and certain drugs.

7. Make the day the routine of life - don't try to catch up on Sundays only.

IV. THE LAST FACTOR in the cure of this national health decline is that of providing for the cases of sickness which occur. It is THE RELIEF SCHEME. Just as we have accident insurance in order to compensate for accidents we should have sickness insurance in order to compensate for sickness. It is best to call this "HEALTH INSURANCE" for we wish to maintain health. Such insurance is the real active agent in bringing persons to observe the Laws of Health, to have Physical Examinations made and to see that their Surroundings are Healthful. To bring this about the insurance should be supported by the worker $(2 / 5)$, by the employer $(2 / 5)$, and by the state $(1 / 5)$. By this means every one is financially interested in prolonging health, increasing efficiency and maintaining a high standard of production. 
III. AVOID DANGERS TO HEALTH - Know What They Are. Here are some of them and their limits:

1. The AIR in most buildings heated by stoves, furnaces, steam or hot water is stagnant, too hot and too dry - dryer than that of the Sahara Desert. Hence sore throats, colds, and many forms of sickness.

GOOD AIR:

CIRCULATES - and by so doing tones up the human system by striking the skin, thus improving the blood-flow, and removing the "envelope of heat" which is given off by the body and constantly surrounds it. Use electric fans or cloth-windows or window-boards or ventilators. A just perceptible "breath" is enough (or air blowing 5 to 10 feet per second).

Has RELATIVE HUMIDITY of 45 to $65 \%$. L,earn the use of the wetbulb thermometer. When not too cold, proper moisture can be had by admitting outdoor air (note cloth-windows). It may take 10 to 20 gallons of water per day for a six-room house. Few, if any, pan and water schemes will evaporate this amount of water.

Has a TEMPERATURE (indoors) of from 60 to $68^{\circ} \mathrm{F}$. This temperature feels perfectly comfortable when the air is properly moistened - it also saves on the coal bill.

Has no irritating FLOATING PARTICLES - dust, smoke, etc.

Has no noticeable GASES, FUMES OR VAPORS - these are especially dangers of modern industries. Any ODOR should be pleasant - the air should be just like that supplied by Nature.

Not POLLUTED by disease germs. Most so-called "air-borne diseases" are really caught by contact with, or close approach to, persons carrying the germs. Such "carriers" may not themselves be sick and also may be in total ignorance of their own menace. Avoid touching persons as much as possible. Keep beyond the breathing range, and, finally, wash your hands often to prevent carrying disease to your face and mouth.

\section{GOOD ILLUMINATION means:}

STEADY LIGHT from over the shoulders - 5-10 foot-candles of ARTIFICIAL light for ordinary eye work, $5 \mathrm{f}$. c. in foundries and similar p!aces, and $3 \mathrm{f}$.c. on the floors of general workrooms. Daylight COLOR is the best. DAYLIGHT should be three times stronger than artificial light. (A foot-candle is the amount of light given by a standard candle one foot away).

Absence of sharp contrasts of lights and shadows; have light-colored walls and ceilings to reflect light instead of absorbing it; unobstructed light, as from the upper half of windows - dirty windows may obstruct $4 / 5$ of the light, while dark shades should not be used in workrooms, offices, or schools; protection of the eyes against brilliant and hot lights.

GOOD EYE-SIGHT - perhaps, after all, your eye-sight is defective and your eyes need examining - most headaches are from eyestrain - eyestrain may be as fatiguing, also, as the most arduous work.

3. POISONS. Workers with poisons should be properly instructed in their use. The COURTS have decided that the responsibility of such instruction rests upon the employer. In Ohio the State Department of Health stands prepared to issue INSTRUCTIONS for the avoidance of poisoning of all sorts. Most poisonings occur because employers and workers both CONSIDER the substances dealt with as too innocent to cause much harm. This attitude has unfortunately caused an immense amount of sickness. numerous deaths, and many LAW SUITS for large amounts of money. The State Laboratories will analyze suspected poisons used in connection with work, provided the sources, uses and reasons for the analyses are stated. The Department of Health will also supply INFORMATION as to the maximum amount of poisonous substances which can be present in the air or may be taken in to the system without causing symptoms - This insofar as it has information or can secure the same. By law, all instances of poisoning in connection with work must be brought TO THE NOTICE of the State Department of Health, which Department furnishes the proper blanks for recording the information and investigates the occurrence with a view to finding out how to avoid similar mishaps. Such reports cannot be used as evidence of the facts therein in any action arising out of the disease 
therein reported. Most poisonings occur through workers poisoning themselves, which is due to IGNORANCE or DISREGARD of instructions, but upon the employer rests the responsibility of supervision. The more COMMON POISONS used in the State of Ohio are about in order of their frequency of use and their liability to produce occupational disease: lead, benzine and benzol (naphtha, petrol, gasoline, etc.), turpentine and similar dryers, brass or zinc in the form of fumes; acids, alkalis, wood alcohol, anilin oil, carbon bisulphide, antimony, illuminating and fuel gas, sulphurated hydrogen, arsenic, phosphorus and mercury.

4. FATIGUE. A tired feeling is nature's warning to rest. Getting tired depends largely upon who you are and how well you may be. Work should promote health and a sense of well-being. "So tired" should never be heard at the end of a day's work, as it signifies exhaustion. All straining or heavy or rapid work should be tempered or subjected to changes so that no feeling of exhaustion results. This applies to the eyes, or to the hands, as well as to the whole body. True "Scientific Management" observes these principles and makes use of several methods of doing routine work. On the other hand, TOO LITTLE EXERCISE is a serious danger to large numbers of persons who are not actively employed. Variation, which will allow exercise, is the secret of efficiency and steadfastness. The best standard for fatigue or inactivity is that "tired feeling". Avoid it.

5. TEMPERATURE. The exposure to heat produces thermic fever if the temperature goes beyond that of the human body $\left(98.6^{\circ}\right)$; beyond this, prostrations, muscular cramps and anemia are produced. Premature old age is a common result. When COMBINED WITH MOISTURE the effects upon the health are very much worse. For the best work, neither the wet nor dry thermometer should exceed $70^{\circ} \mathrm{F}$. The shower-bath is a most excellent way to control the circulation of the blood upon quitting the hot workplace preparatory to going outside. In almost all trades heat can be controlled or kept away from workmen. EXPOSURE TO COLD, pure and simple, is not dangerous to health provided enough clothing is worn and enough active work is performed. Cooling one part of the body while heating another is bad.

6. DIRT AND DISORDERED SURROUNDINGS are dangerous to health because they favor disease, obstruct light, make one less inclined to do a high class of work; also to observe health standards, correct habits and good morals, whether at home, at work, or elsewhere.

7. THE NATURAL DESIRES create great risks to health -

1. THIRST must be supplied with safe drinking water.

2. HUNGER must be supplied with clean, nourishing food eaten in a place free from poisons.

3. TOILETS, URINALS, SEWERAGE and GARBAGE devices must not engender disease.

4. SLEEP must not be disturbed and must last eight hours every night.

5. RECREATION must not be dissipative but recreative.

6. The desire for STIMULANTS is always abnormal to the healthy person; when present it is usually due to the effects of some of the dangers above cited or to a habit acquired through ignorance or bravado. Stimulants include alcohol, coffee, tea and certain drugs.

7. Make the day the routine of life - don't try to catch up on Sundays only.

IV. THE LAST FACTOR in the cure of this national health decline is that of providing for the cases of sickness which occur. It is THE RELIEF SCHEME. Just as we have accident insurance in order to compensate for accidents we should have sickness insurance in order to compensate for sickness. It is best to call this "HEALTH INSURANCE" for we wish to maintain health. Such insurance is the real active agent in bringing persons to observe the Laws of Health, to have Physical Examinations made and to see that their Surroundings are Healthful. To bring this about the insurance should be supported by the worker $(2 / 5)$, by the employer $(2 / 5)$, and by the state $(1 / 5)$. By this means every one is financially interested in prolonging health, increasing efficiency and maintaining a high standard of production. 



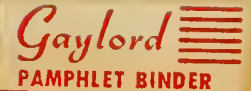

$\equiv$ Syracuse, N. Y. 
